\title{
Radiation Absorption and Variable Electrical Conductivity Effect on High Speed MHD Free Convective Flow past an Exponential Accelerated Inclined Plate
}

\author{
B. M. Jewel Rana, Raju Roy, Lasker Ershad Ali, S. F. Ahmmed \\ Mathematics Discipline Khulna University, Khulna, Bangladesh \\ Email: Jewelrana.ku@gmail.com
}

How to cite this paper: Jewel Rana, B.M., Roy, R., Ali, L.E. and Ahmmed, S.F. (2017) Radiation Absorption and Variable Electrical Conductivity Effect on High Speed MHD Free Convective Flow past an Exponential Accelerated Inclined Plate. World Journal of Mechanics, 7, 211-241.

https://doi.org/10.4236/wjm.2017.78019

Received: June 22, 2017

Accepted: August 17, 2017

Published: August 20, 2017

Copyright $\odot 2017$ by authors and Scientific Research Publishing Inc. This work is licensed under the Creative Commons Attribution International License (CC BY 4.0).

http://creativecommons.org/licenses/by/4.0/ (c) † Open Access

\begin{abstract}
The effects of variable electrical conductivity and radiation absorption on high-speed MHD (Magnetohydrodynamics) free convective flow past an exponential accelerated inclined plate with higher order chemical reaction have been investigated. The electrical conductivity has been considered as a function of temperature. The chemical reaction has also been considered as the higher order. The usual transformations have been used to obtain the coupled dimensionless momentum, energy and concentration equations. Then the obtained non-dimensional partial differential equations have been solved numerically by explicit finite difference method. For excellent accuracy, stability and convergence test have been carried out. A computer program Compaq Visual FORTRAN 6.6a has been used to calculate the numerical results. The obtained numerical results concerned with the fluid velocity, temperature, concentration, concerned with skin friction, Nusselt number, Sherwood number, streamlines and isotherms are imprinted graphically by Tecplot and discussed in details. It is noticed that increasing of magnetic parameter increases the temperature and decreases the velocity, skin friction and Nusselt number. It is also observed that the temperature profile is increasing with the increasing values of Dufour number. However, the order of the chemical reaction increases the concentration profile.
\end{abstract}

\section{Keywords}

Radiation Absorption, Thermal Radiation, Variable Electrical Conductivity, Variable Temperature, Higher Order Chemical Reaction 


\section{Introduction}

A fluid is a substance that is subject to deformation upon application of any shear force or stress. Fluid dynamics is an area of study that deals with the flow of fluids. The fluids in consideration include gases, liquids and ionized gases are also called plasma. Electromagnetism on the other hand studies the interaction between the electric and magnetic fields. Magnetohydrodynamics (MHD) is the study of motion of electrically conducting fluids with the influence of electromagnetic phenomena. Magnetohydrodynamics is now undertaking a stage of great development. The MHD in the current form is due to contributions of several notable authors like Shercliff [1], Ferraro and Plumpton [2], Jaffrey [3] and Pop and Ingham [4]. There are numerous engineering applications for this phenomenon, such as solar structures, astrophysics, purification of crude oil, underground water storage system, soil sciences, paper industry, textile industry, pulp, nuclear power reactor, accelerators in geophysics and design of MHD generators. The applications of MHD principles in medicine and biology are of salient interest owing to their importance in biomedical engineering such as cardiac MRI, ECG etc.

Convection flows have greatly fascinated the researchers and engineers due to their presence both in nature and engineering applications. Free convection refers to the motion of fluid initiated exclusively owing to the density difference during the cooling or heating of the fluid. It might also arise due to the difference in both temperature gradients and species concentration. The study of free convection flow has many momentous applications such as the nuclear reactor, thermal hydraulics, frost formation and channel-chimney systems. Laminar free convection has many industrial and environmental applications such as thermal regulation process, motors, atmospheric flows, human comfort in buildings, and electronic cooling devices. A free convective heat transfer problem was first performed by Finston [5]. The first elementary assumptions of fluid dynamics are the conservation of mass or the continuity of mass with a flow system. The second elementary assumptions of fluid dynamics are the conservation of linear momentum or Newton's second law of motion. The third elementary assumptions of fluid dynamics are the conservation of energy or the first law of thermo-dynamics.

A fundamental law linking pressure drop and velocity in fluid flow through porous media is Darcy's law. The flow of a fluid through a porous medium is delineated by the Darcy's law.

With the experimental results on the flow of water through beds of sand, Henry Darcy was initiated the Darcy's law. Darcy's law is employed to scrutinize water flow through an aquifer. Also Darcy's law is applied to recount gas, water, and oil flows through petroleum reservoirs. The Darcy linear model is insufficient in the presence of higher velocities. In this case an inertial term is added to the Darcy's equation, which is known as Forchheimer term. Forchheimer [6] investigated fluid flow through porous media in the high velocity regime. $\mathrm{He}$ 
observed that as the flow velocity increases, the inertial effects start dominating the flow. In order to account for these high velocity inertial effects, he prescribed the inclusion of an inertial term representing the kinetic energy of the fluid to the Darcy equation. Apparently the most popular modification to Darcian flows is the Darcy Forchheimer model. Many scholars have modified Darcy's law such as Wooding [7] and Brinkman [8] [9] to study the convective flow in porous media accurately.

When the solid boundary permits fluid to pass through it, then we can consider a porous medium. A porous medium is said to be homogenous if the ratio of the pore area to the total area of the solid boundary is a constant. Otherwise the medium is termed as non-homogeneous. Porous media can be used as an insulator and also can be utilized as a heat transfer promoter for either sensible or latent heat transfer. The heat transfer flow through a porous media has empiric applications particularly in geophysical fluid dynamics such as beach sand, sandstone, limestone, wood, small blood vessels, and in the human lung. Free convection with the porous medium has various technological applications such as porous material regenerative heat exchangers, energy storage, oil extraction, cool combustors, granular insulation, electronic system cooling, fiber, and fluid flow through filtering devices. The coupled mass and heat transfer arise in the fluid owing to buoyancy forces generated by concentration difference and temperature difference. Coupled heat and mass transfer in porous media has momentous applications such as underground energy transport, enhanced oil recovery, cooling of nuclear reactors and geothermal reservoirs. MHD free convective, dissipative boundary layer flow past a porous vertical surface in the presence of thermal radiation, chemical reaction and constant suction have been investigated by Raju et al. [10] using a regular perturbation technique.

Thermal radiation is a process by which energy is emitted by a heated surface in all directions in the form of electromagnetic radiation and travels in a combination of magnetic and electric waves. Recently, Mondal et al. [11] have studied the effect of radiation on heat transfer problems. The study of thermal radiation on free convection flow has various practical applications such as high-speed flights, re-entry of space vehicle and power generation plants. The influence of radiation on MHD flow with heat and mass transfer plays a vital role in industrial and technological areas such as various space vehicles, various propulsion devices for satellites, missiles, aircraft, gas turbines and nuclear power plants. Radiation and mass transfer effects on unsteady MHD convective flow past an infinite vertical plate with Dufour and Soret effects have been delineated by Vedavathi et al. [12]. In their study, they have made use of shooting method along with Runge-Kutta fourth order integration technique. Prakash et al. [13] have applied perturbation technique to investigate Diffusion-thermo effects on MHD free convective radiative and chemically reactive boundary layer flow through a porous medium over a vertical plate. MHD free convection flow of a non-Newtonian fluid past an impulsively started vertical plate in the presence of thermal 
diffusion and radiation absorption has been explicated by Umamaheswar et al. [14]. From the observation of Vedavathi et al. [12], Prakash et al. [13] and Umamaheswar et al. [14], it is noticed that the velocity and temperature in the boundary layer increase owing to the presence of absorption of radiation.

The free convection problem in the presence of high-speed magnetic field has enormous industrial applications such as welding, the safety aspect of gas-cooled reactors, solar energy collectors, crystal growth, materials processing and cooling reactors. Desale and Pradhan [15] have used implicit finite difference technique to obtain the solution of MHD boundary layer heat transfer over a moving plate. Mishra and Jena [16] have used Runge-Kutta fourth-order algorithm to calculate the numerical results of boundary layer MHD flow with viscous dissipation and the computations have been done by a computational computer language, MATLAB. Haque and Sarder [17] have imposed explicit finite difference scheme to complete the investigation of convective heat and mass transfer of high-speed MHD flow over a stretching sheet with the influence of thermal diffusion. Haque and Sarder [17] have also discussed the stability criteria of the explicit finite difference method in a nutshell. From the experiments of Desale and Pradhan [15], Mishra and Jena [16] and Haque and Sarder [17], it is observed that temperature enhances in the presence of high-speed MHD.

Dissipation is the strait of metamorphosing mechanical energy of downward-flowing water into acoustical and thermal energy. Viscous dissipation effects play a momentous role in free convection in various devices which are operated at high speeds. To define the viscosity of dilute suspensions, dissipation function is used (Einstein [18]). Viscous dissipation with magneto hydrodynamic flow has many momentous geothermal, technological and industrial applications such as MHD accelerators and MHD power generation, liquid, and metal nuclear reactors. When the flow field is in high gravitational field or at low temperature or of exhaustive size, the viscous dissipation heat in the free convective flow is momentous. The importance of viscous dissipative heat in free convection flow in the case of constant heat flux and isothermal at the plate has been demonstrated by Gebharat [19]. Variable heat and mass transfer under the assumption of different physical phenomena have already been carried out by many scholars. Kishore et al. [20] have employed implicit finite difference method of Crank-Nicolson's type to obtain the numerical solution of viscous dissipation and heat transfer on MHD free convection flow past an exponentially accelerated vertical plate with variable temperature. They have also analyzed stability and convergence test of implicit finite difference method. The flow of an electrically conducting fluid past a continuously moving plate was initiated by Sakiadis [21].

Abd El-Naby et al. [22] have used finite difference scheme to get the solution of radiation effects on MHD unsteady free-convection flow over the vertical plate with variable surface temperature. In their investigation, they have applied the von Neumann method to study the stability criterion for the finite difference 
technique. Rajput and Kumar [23] have studied radiation effects on MHD flow past an impulsively started vertical plate in the presence of variable heat and mass transfer with the help of Laplace transformation technique. Unsteady Magnetohydrodynamics free convective flow past a vertical porous plate has been presented by Gundagani et al. [24] by employing a finite element method. Javaherdeh et al. [25] have imposed implicit finite difference method to find the numerical solution of MHD free convection heat and mass transfer flow past a moving vertical plate in a porous medium. The temperature and concentration level at the plate surface have been followed a power law type of distribution in their observation. Pattnaik et al. [26] have investigated mass transfer and radiation effects on MHD flow through porous medium past an exponentially accelerated inclined plate with variable temperature. The Laplace transformation method has been used to solve the governing equations in the computations of Pattnaik et al. [26]. From the analysis of Pattnaik et al. [26], it is reported that the presence of magnetic field and angle of inclination prevents the flow reversal.

The effect of variable electrical conductivity has not been taken into consideration, in the above pointed out investigations. Engineers can control many metallurgical processes by taking suitable electrical conductivity of fluids. Steady MHD natural convection flow with variable electrical conductivity and heat generation along an isothermal vertical plate has been studied by Shrama and Singh [27] by applying Runge-Kutta fourth order method along with shooting iteration scheme. The authors have used similarity transformation for converting partial differential equations into ordinary differential equations. Also, the authors have used commercial software Mathematica for drawing graphs. Chakraborty and Medhi [28] have investigated MHD convective flow along a vertical isothermal plate under variable electrical conductivity and heat generation in a porous medium by using Runge-Kutta method of shooting iteration technique. They have considered that the electrical conductivity is a function of temperature and the Prandtl number is very low.

The rate of mass diffusion can be changed effectively by the presence of chemical reactions. In nature it is quite improbable to find pure fluid unless special efforts are taken to get it. The most common fluids like water and air are contaminated with impurities like $\mathrm{CO}_{2}, \mathrm{C}_{6} \mathrm{H}_{6}$ and $\mathrm{H}_{2} \mathrm{SO}_{4}$ etc. When such contaminant is contain in the fluid under consideration there takes place some chemical reaction e.g. water and sulfuric acid react chemically, also air and benzene (Umamaheswar et al. [14]). In most of cases of chemical reaction, the reaction rate counts on the concentration of the species itself. Heat will be originated when a chemical reaction takes place between two species. We can take a first order chemical reaction when the foreign mass exists in the fluid at very low lev$\mathrm{el}$ and the heat originated owing to the chemical reaction can be ignored (Sharma and Deka [29]). If the rate of reaction is directly proportional to concentration itself, then the reaction is said to be of first-order (Sharma et al. [30]). A reaction is said to be of the order $l$, if the reaction rate is proportional to the 
l-power of concentration. Chemical reaction can be classified as either heterogeneous or homogeneous processes. The reaction is homogeneous if it occurs in solution and heterogeneous if it occurs at an interface in a well mixed system. The combined heat and mass transfer problems in the presence of higher order chemical reaction are important in chemical industries like in curing of plastics, food processing, manufacture of pulp, insulated cables and polymer production. It is significant to investigate the effects of heat generation or absorption when fluids undertaking endothermic or exothermic chemical reactions. The presence of heat generation or absorption can be used in semiconductor wafers and electronic chips. Alam and Ahammad [31] have applied Nachtsheim-Swigert shooting iteration technique with sixth-order Runge-Kutta integration scheme to study the effects of variable chemical reaction and variable electric conductivity on free convective heat and mass transfer flow along an inclined stretching sheet with variable heat and mass fluxes under the influence of Dufour and Soret effects. They have considered that electrical conductivity is a function of velocity. They have observed that velocity and concentration profiles increase but temperature profiles decrease with the increasing values of order of chemical reaction. MHD free convective flow over a vertical cone with variable electric conductivity in the presence of chemical reaction has been delineated by Kumar et al. [32] by applying an efficient finite difference technique of the Crank-Nicolson type.

In the above study, most of the scholars have used $P_{r}=0.71$ and $S_{c}=0.60$ in their computations. The ultimate aim of the this present problem is to develop the effects of variable electrical conductivity and radiation absorption on high speed MHD fee convective flow past an exponential accelerated inclined plate. The electrical conductivity has been taken as a function of temperature. The chemical reaction has also been assumed as higher order. From Alam and Ahammad [31] it is noticed that the reaction having order four or more are practically improbable. Therefore we have also considered the order of the reaction as $I=1 ; 2$ and 3 in this research investigation. The coupled partial differential equations are transformed into dimensionless by applying non-dimensional quantities. Then the resultant dimensionless equations are solved numerically by employing an efficient, accurate and conditionally stable finite difference technique of explicit type with the help of a program FORTRAN 6.6a. The stability and convergence analysis has been carried out to establish the effect of velocity, temperature, concentration, skin friction, Nusselt number and Sherwood number. At last, the effects of momentous parameters are presented graphically and discussed qualitatively.

\section{Mathematical Formulation}

Consider an unsteady two-dimensional laminar free convective flow past a porous plate, which is embedded in an incompressible viscous fluid having temperature dependent electrical conductivity. The fluid is taken to be a gray, absorbing 
emitting radiation but not scattering the medium. The plate is inclined to the vertical direction by an angle $\varphi$. The $x$-axis is directed along the plate in the direction of the fluid and $y$-axis is considered normal to the plate in the fluid. At first, it is taken that the plate and the fluid are of the same temperature $T_{\infty}$ with concentration level $C_{\infty}$ at all points. At time $t>0$, the plate is exponentially accelerated in its own plane with a velocity $U_{o} \exp \left(a^{*} t\right)$. At the same time, the temperature of the plate and the concentration level are also decreased or increased exponentially with respect to time $t$. It is considered that a radiative heat flux $q_{r}$ is employed in the perpendicular direction to the plate. It is also considered that a higher order chemical reaction taking place between the fluid and the diffusing species. A transverse magnetic field of fixed magnitude $B_{o}$ is employed perpendicular to the direction of the flow. It is considered that the induced magnetic field is insignificant in comparison to the employed magnetic field. The physical diagram of the problem is elucidated in Figure 1.

Under the boundary layer approximation, governing equations that deliberate the physical condition of the problem are given by

$$
\begin{gathered}
\frac{\partial u}{\partial x}+\frac{\partial v}{\partial y}=0 \\
\frac{\partial u}{\partial t}+u \frac{\partial u}{\partial x}+v \frac{\partial u}{\partial y}=v \frac{\partial^{2} u}{\partial y^{2}}+g \beta^{\prime}\left(T-T_{\infty}\right) \cos \varphi+g \beta^{*}\left(C-C_{\infty}\right) \cos \varphi \\
-\frac{\sigma^{\prime} B_{o}^{2} u}{\rho}-\frac{v}{k} u-\frac{b}{k} u^{2} \\
\frac{\partial T}{\partial t}+u \frac{\partial T}{\partial x}+v \frac{\partial T}{\partial y}=\frac{\kappa}{\rho c_{p}} \frac{\partial^{2} T}{\partial y^{2}}-\frac{1}{\rho c_{p}} \frac{\partial q_{r}}{\partial y}+\frac{D_{m} k_{T}}{C_{s}} \frac{\partial^{2} C}{\partial y^{2}}+\frac{Q_{o}}{\rho c_{p}}\left(T-T_{\infty}\right) \\
+\frac{Q_{1}^{*}}{\rho c_{p}}\left(C-C_{\infty}\right)+\frac{\mu}{\rho c_{p}}\left(\frac{\partial u}{\partial y}\right)^{2}+\frac{\sigma^{\prime} B_{o}^{2} u^{2}}{\rho c_{p}}
\end{gathered}
$$

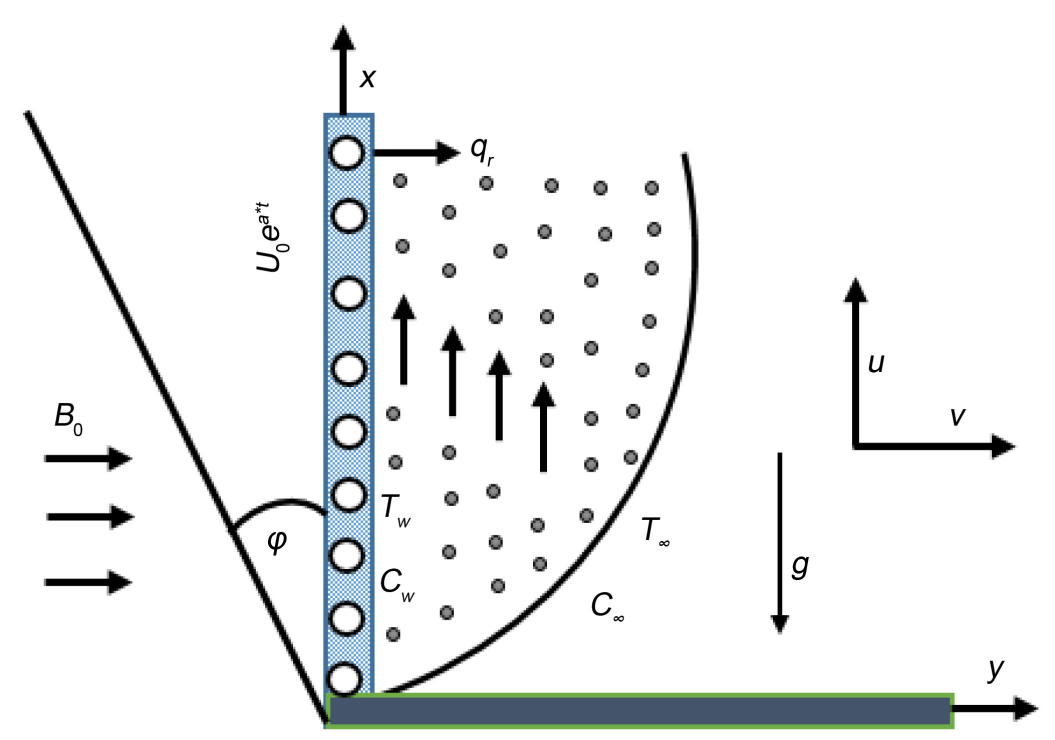

Figure 1. Physical model and coordinate system. 


$$
\frac{\partial C}{\partial t}+u \frac{\partial C}{\partial x}+v \frac{\partial C}{\partial y}=D_{m} \frac{\partial^{2} C}{\partial y^{2}}+\frac{D_{m} k_{T}}{T_{m}} \frac{\partial^{2} T}{\partial y^{2}}-k_{r}\left(C-C_{\infty}\right)^{l}
$$

where $x$ and $y$ are the dimensional distance along and perpendicular to the plate respectively. $u$ and $v$ are the velocity components in the $x$ and $y$ directions respectively, $t$ is the time, $v$ is the kinematics viscosity, $\mu$ is the viscosity, $g$ is the gravitational acceleration, $\sigma$ is the electric conductivity of the fluid in the boundary layer region depending on the fluid temperature, $B_{o}$ is the magnetic induction, $\rho$ is the fluid density, $\beta^{\prime}$ and $\beta^{*}$ are the heat and mass transfer volume expansion coefficients respectively, $k$ is the Darcy permeability, $b$ is the empirical constant (Vedavathi et al. [8]), $\kappa$ is the thermal conductivity, $c_{p}$ is the specific heat at constant pressure, $c_{s}$ is the concentration susceptibility, $D_{m}$ is the molecular diffusivity of the species concentration, $Q_{1}^{*}$ is the coefficient of proportionality of the radiation, $T_{m}$ is the mean fluid temperature, $\kappa_{T}$ is the thermal diffusion ratio, $a^{*}$ is the dimensional acceleration parameter, $k_{r}$ is the reaction rate constant, $l$ is the order of chemical reaction and $Q_{o}$ is the heat generation constant, which may be either positive or negative. The heat source becomes positive in case of heat generation and negative in case of heat absorption.

The initial and boundary conditions relevant to the present problem are as follows:

$$
\left.\begin{array}{rl}
t \leq 0: u & \left.=0, v=0, T=T_{\infty}, C=C_{\infty}\right\} \text { for all } y \\
t>0: u & =U_{\circ} \exp \left(a^{*} t\right), v=0, T=T_{\infty}+\left(T_{w}-T_{\infty}\right) \exp \left(\frac{U_{\circ}^{2}}{v} t\right), \\
C & =C_{\infty}+\left(C_{w}-C_{\infty}\right) \exp \left(\frac{U_{\circ}^{2}}{v} t\right) \\
u & \left.=0, T \rightarrow T_{\infty}, C \rightarrow C_{\infty}\right\} \text { as } y \rightarrow \infty
\end{array}\right\}
$$

A few forms of electrical conductivity difference are found in the literature. Among them we have taken that one which is absolute for liquid as follows:

$$
\frac{1}{\sigma^{\prime}}=\frac{1}{\sigma}\left[1+\varepsilon^{\prime}\left(T-T_{\infty}\right)\right]
$$

where $\sigma$ is the electrical conductivity at the surface temperature and $\varepsilon^{\prime}$ is a constant based on the electrical property of the fluid.

Here we introduce a non-dimensional electrical conductivity variation parameter $\varepsilon$ such that

$$
\varepsilon=\varepsilon^{\prime}\left(T_{w}-T_{\infty}\right)
$$

Substituting variable (7) into (6) takes to the following form

$$
\sigma^{\prime}=\frac{\sigma}{1+\varepsilon \theta}
$$

A similar result (8) has been presented by Shrama and Singh [27]

The radiative heat flux for the matter of an optically thin gray gas can be written as (Raju et al. [10]) 


$$
q_{r}=-\frac{4 \sigma^{*}}{3 k_{1}} \frac{\partial T^{4}}{\partial y}
$$

where $\sigma^{*}$ and $k_{1}$ are the Stefan-Boltzmann constant and mean absorption coefficient respectively. We considered that the temperature changes within the flow are adequately small such that $T^{4}$ may be expanded as a linear function of the temperature. Expanding $T^{4}$ in a Taylor series about $T_{\infty}$ and ignoring higher order terms, we obtain

$$
T^{4} \cong 4 T_{\infty}^{3} T-3 T_{\infty}^{4}
$$

Substituting Equation (10) into (9) leads to the following form

$$
q_{r}=-\frac{16 \sigma^{*} T_{\infty}^{3}}{3 k_{1}} \frac{\partial T}{\partial y}
$$

The non-dimensional variables appropriate to this problem are as follows:

$$
\begin{aligned}
& X=\frac{x U_{\circ}}{v}, Y=\frac{y U_{\circ}}{v}, U=\frac{u}{U_{\circ}}, V=\frac{v}{U_{\circ}}, \tau=\frac{t U_{\circ}^{2}}{v}, \theta=\frac{T-T_{\infty}}{T_{w}-T_{\infty}}, \phi=\frac{C-C_{\infty}}{C_{w}-C_{\infty}}, \\
& \left.\begin{array}{l}
a=\frac{a^{*} v}{U_{\circ}^{2}}, G_{r}=\frac{g \beta v\left(T_{w}-T_{\infty}\right)}{U_{\circ}^{3}}, G_{m}=\frac{g \beta^{*} v\left(C_{w}-C_{\infty}\right)}{U_{\circ}^{3}}, M=\frac{\sigma B_{\circ}^{2} v}{\rho U_{\circ}^{2}}, D_{a}=\frac{k U_{\circ}^{2}}{v^{2}}, \\
F_{s}=\frac{b U_{\circ}}{v}, P_{r}=\frac{\mu c_{p}}{k}, R=\frac{4 \sigma^{*} T_{\infty}^{3}}{k^{*} k}, Q=\frac{Q_{\circ} v}{\rho c_{p} U_{\circ}^{2}}, Q_{1}=\frac{Q_{1}^{*} v}{\rho c_{p} U_{\circ}^{2}}, E_{c}=\frac{U_{\circ}^{2}}{c_{p}\left(T_{w}-T_{\infty}\right)}, \\
D_{u}=\frac{D_{m} k_{T}}{v c_{s} c_{p}}\left(\frac{C_{w}-C_{\infty}}{T_{w}-T_{\infty}}\right), S_{c}=\frac{v}{D_{m}}, S_{r}=\frac{D_{m} k_{T}}{v T_{m}}\left(\frac{T_{w}-T_{\infty}}{C_{w}-C_{\infty}}\right), K_{c}=\frac{k_{r} v\left(C_{w}-C_{\infty}\right)^{l-1}}{U_{0}^{2}}
\end{array}\right\}
\end{aligned}
$$

Insertion of Equations (12), (11) and (8) into (1)-(4) leads to the following dimensionless equations:

$$
\begin{gathered}
\frac{\partial U}{\partial X}+\frac{\partial V}{\partial Y}=0 \\
\frac{\partial U}{\partial \tau}+U \frac{\partial U}{\partial X}+V \frac{\partial U}{\partial Y}=\frac{\partial^{2} U}{\partial Y^{2}}+G_{r} \cos \varphi+G_{m} \cos \varphi-\left(\frac{M}{1+\varepsilon \theta}+\frac{1}{D_{a}}\right) U-\frac{F_{s}}{D_{a}} U^{2} \\
\frac{\partial \theta}{\partial \tau}+U \frac{\partial \theta}{\partial X}+V \frac{\partial \theta}{\partial Y}=\frac{1}{P_{r}}\left(1+\frac{4}{3} R\right) \frac{\partial^{2} \theta}{\partial Y^{2}}+D_{u} \frac{\partial^{2} \phi}{\partial Y^{2}}+Q \theta+Q_{1} \phi+E_{c}\left(\frac{\partial U}{\partial Y}\right)^{2}+E_{c}\left(\frac{M}{1+\varepsilon \theta}\right) U^{2} \\
\frac{\partial \phi}{\partial \tau}+U \frac{\partial \phi}{\partial X}+V \frac{\partial \phi}{\partial Y}=\frac{1}{S_{c}} \frac{\partial^{2} \phi}{\partial Y^{2}}+S_{r} \frac{\partial^{2} \theta}{\partial Y^{2}}-K_{c} \phi^{l}
\end{gathered}
$$

The corresponding initial and boundary conditions are as follows:

$$
\left.\begin{array}{c}
\tau>0: U=\exp (a \tau), V=0, \theta=\exp (\tau), C=\exp (\tau)\} \text { at } Y=0 \\
U \rightarrow 0, \theta \rightarrow 0, \phi \rightarrow 0\} \text { as } Y \rightarrow \infty
\end{array}\right\}
$$

The parameters of technical attraction for the present problem are the local skin-friction, the local Nusselt number and the local Sherwood number, which elucidates physically the wall shear stress, the rate of heat transfer and the rate of mass transfer respectively. Furthermore it is important to note that the coefficient of skin friction, Nusselt number and Sherwood number can be obtained by 
the analysis of velocity, temperature and concentration field. The dimensionless forms of skin-friction, Nusselt number and Sherwood number are as follows:

$$
\begin{gathered}
C_{f}=-\frac{1}{2 \sqrt{2}} G_{r}^{-\frac{3}{4}}\left(\frac{\partial U}{\partial Y}\right)_{Y=0} \\
N_{u}=\frac{1}{\sqrt{2}} G_{r}^{-\frac{3}{4}}\left(\frac{\partial \theta}{\partial Y}\right)_{Y=0} \\
S_{h}=\frac{1}{\sqrt{2}} G_{r}^{-\frac{3}{4}}\left(\frac{\partial \phi}{\partial Y}\right)_{Y=0}
\end{gathered}
$$

\section{Numerical Solution}

The present problem has required a set of finite difference equations. In this process the region within the boundary layer is divided into grid or meshes of lines parallel to $X$ and $Y$ coordinates where $X$-axis is considered along the plate and $Y$-axis is perpendicular to the plate. Here the plate of height is taken $X_{\max }$ $(=20)$ i.e. $X$ varies from 0 to 20 and as corresponds to $Y_{\max }(=25)$ i.e. $Y$ varies from 0 to 25. Grid spacing in the $X$ and $Y$ direction are $m_{\max }=100$ and $n_{\max }=$ respectively. $\Delta X$ and $\Delta Y$ are constant mesh size along $X$ and $Y$ directions as follows $\Delta X=0.20(0 \leq X \leq 20)$ and $\Delta Y=0.125(0 \leq Y \leq 25)$ with a smaller time- step $\Delta \tau=0.0002$.

Let $U^{\prime}, \theta^{\prime}$ and $\phi^{\prime}$ indicated the values of $U, \theta$ and $\phi$ at the end of a time-step respectively. Applying the explicit finite difference technique, the following appropriate set of finite difference equations are obtained from the dimensionless coupled partial differential Equations (13)-(16)

$$
\begin{aligned}
& \frac{U_{i, j}-U_{i, j-1}}{\Delta X}+\frac{V_{i, j}-V_{i, j-1}}{\Delta Y}=0 \\
& \frac{U_{i, j}^{\prime}-U_{i, j}}{\Delta \tau}+U_{i, j} \frac{U_{i, j}-U_{i-1, j}}{\Delta X}+V_{i, j} \frac{U_{i, j+1}-U_{i, j}}{\Delta Y} \\
& =\frac{U_{i, j+1}-2 U_{i, j}+U_{i, j-1}}{(\Delta Y)^{2}}+G_{r} \cos \varphi \theta_{i, j}+G_{m} \cos \varphi \phi_{i, j}-\left(\frac{M}{1+\varepsilon \theta_{i, j}}+\frac{1}{D a}\right) U_{i, j}-\frac{F_{s}}{D a}\left(U_{i, j}\right)^{2} \\
& \frac{\theta_{i, j}^{\prime}-\theta_{i, j}}{\Delta \tau}+U_{i, j} \frac{\theta_{i, j}-\theta_{i-1, j}}{\Delta X}+V_{i, j} \frac{\theta_{i, j+1}-\theta_{i, j}}{\Delta Y} \\
& =\frac{1}{P_{r}}\left(1+\frac{4 R}{3}\right)\left(\frac{\theta_{i, j+1}-2 \theta_{i, j}+\theta_{i, j-1}}{(\Delta Y)^{2}}\right)+D_{u}\left(\frac{\phi_{i, j+1}-2 \phi_{i, j}+\phi_{i, j-1}}{(\Delta Y)^{2}}\right) \\
& +Q \theta_{i, j}+Q_{1} \phi_{i, j}+E_{c}\left(\frac{U_{i, j+1}-U_{i, j}}{\Delta Y}\right)^{2}+E_{c}\left(\frac{M}{1+\varepsilon \theta_{i, j}}\right)\left(U_{i, j}\right)^{2} \\
& \frac{\phi_{i, j}^{\prime}-\phi_{i, j}}{\Delta \tau}+U_{i, j} \frac{\phi_{i, j}-\phi_{i-1, j}}{\Delta X}+V_{i, j} \frac{\phi_{i, j+1}-\phi_{i, j}}{\Delta Y} \\
& =\frac{1}{S_{c}}\left(\frac{\phi_{i, j+1}-2 \phi_{i, j}+\phi_{i, j-1}}{(\Delta Y)^{2}}\right)+S_{r}\left(\frac{\theta_{i, j+1}-2 \theta_{i, j}+\theta_{i, j-1}}{(\Delta Y)^{2}}\right)-K_{c}\left(\phi_{i, j}\right)^{l}
\end{aligned}
$$

The associated initial and boundary conditions with the finite difference tech- 
nique are

$$
\left.\begin{array}{rl}
\tau>0: U_{i, 0}^{p} & =\exp (a p \Delta \tau), V=0, \theta=\exp (p \Delta \tau), C=\exp (p \Delta \tau)\} \text { at } Y=0 \\
U_{i, L}^{p} & \left.\rightarrow 0, \theta_{i, L}^{p} \rightarrow 0, \phi_{i, L}^{p} \rightarrow 0\right\} \text { as } Y \rightarrow \infty
\end{array}\right\}
$$

Here the subscripts $i$ and $j$ represent the mesh points with $X$ and $Y$ axes respectively and the superscripts $p$ designates a value of time, $\tau=p \Delta \tau$ where $p=0,1,2,3, \cdots$.

\section{Stability and Convergence Analysis}

The analysis of this present problem will remain incomplete unless we study the stability of explicit finite difference method. The general term of the Fourier expansion for $U, \theta$ and $\phi$ at a time arbitrarily called $\tau=0$, is taken to be of the form $\mathrm{e}^{\mathrm{i} \alpha X} \mathrm{e}^{\mathrm{i} \beta Y}$, where $i=\sqrt{-1}$. At a later time $\tau$, these terms become

$$
\begin{aligned}
& U: F(\tau) \mathrm{e}^{i \alpha X} \mathrm{e}^{i \beta Y} \\
& \theta: G(\tau) \mathrm{e}^{i \alpha X} \mathrm{e}^{i \beta Y} \\
& \phi: H(\tau) \mathrm{e}^{i \alpha X} \mathrm{e}^{i \beta Y}
\end{aligned}
$$

and after the time step the terms of the Fourier expansion for $U, \theta$ and $\phi$ will be:

$$
\begin{aligned}
& U: F^{\prime}(\tau) \mathrm{e}^{i \alpha X} \mathrm{e}^{i \beta Y} \\
& \theta: G^{\prime}(\tau) \mathrm{e}^{i \alpha X} \mathrm{e}^{i \beta Y} \\
& \phi: H^{\prime}(\tau) \mathrm{e}^{i \alpha X} \mathrm{e}^{i \beta Y}
\end{aligned}
$$

Applying the Expressions (26) and (27) into Equations (22)-(24), taking the coefficients $U$ and $V$ as constants over any one time step, we get the following equations upon simplification

$$
\begin{aligned}
& \frac{\left(F^{\prime}-F\right) \mathrm{e}^{i \alpha X} \mathrm{e}^{i \beta Y}}{\Delta \tau}+U \frac{F \mathrm{e}^{i \alpha X} \mathrm{e}^{i \beta Y}\left(1-\mathrm{e}^{-i \alpha \Delta X}\right)}{\Delta X}+V \frac{F \mathrm{e}^{i \alpha X} \mathrm{e}^{i \beta Y}\left(\mathrm{e}^{i \beta \Delta Y}-1\right)}{\Delta Y} \\
= & G_{r} G \mathrm{e}^{i \alpha X} \mathrm{e}^{i \beta Y} \cos \varphi+G_{m} H \mathrm{e}^{i \alpha X} \mathrm{e}^{i \beta Y} \cos \varphi+\varepsilon M G \mathrm{e}^{i \alpha X} \mathrm{e}^{i \beta Y} U \\
& +\frac{2 F \mathrm{e}^{i \alpha X} \mathrm{e}^{i \beta Y}(\cos \beta \Delta Y-1)}{(\Delta Y)^{2}}-\left(M+\frac{1}{D_{a}}\right) U-\frac{F_{s}}{D_{a}} U^{2},
\end{aligned}
$$

The Equations (28)-(30) can be written in the following form

$$
\begin{gathered}
F^{\prime}=A_{1} F+A_{2} G+A_{3} H \\
G^{\prime}=A_{4} F+A_{5} G+A_{6} H \\
H^{\prime}=A_{7} G+A_{8} H
\end{gathered}
$$

where

$$
\begin{gathered}
A_{1}=1+\frac{2 \Delta \tau(\cos \beta \Delta Y-1)}{(\Delta Y)^{2}}-\left(M+\frac{1}{D_{a}}\right) \Delta \tau-\frac{F_{s}}{D_{a}} U \Delta \tau \\
-U \frac{\Delta \tau\left(1-\mathrm{e}^{-i \alpha \Delta X}\right)}{\Delta X}-V \frac{\Delta \tau\left(\mathrm{e}^{i \beta \Delta Y}-1\right)}{\Delta Y} \\
A_{2}=G_{r} \Delta \tau \cos \varphi+\varepsilon M U \Delta \tau
\end{gathered}
$$




$$
\begin{gathered}
A_{3}=G_{m} \Delta \tau \cos \varphi \\
A_{4}=E_{c} M U \Delta \tau+E_{c} U \Delta \tau\left(\frac{\mathrm{e}^{i \beta \Delta Y}-1}{\Delta Y}\right)^{2} \\
A_{5}=1+\frac{1}{P_{r}}\left(1+\frac{4}{3} R\right) \frac{2 \Delta \tau(\cos \beta \Delta Y-1)}{(\Delta Y)^{2}}+Q \Delta \tau-E_{c} M \varepsilon U^{2} \Delta \tau \\
-U \frac{\Delta \tau\left(1-\mathrm{e}^{-i \alpha \Delta X}\right)}{\Delta X}-V \frac{\Delta \tau\left(\mathrm{e}^{i \beta \Delta Y}-1\right)}{\Delta Y} \\
A_{6}=D_{u} \frac{2 \Delta \tau(\cos \beta \Delta Y-1)}{(\Delta Y)^{2}}+Q_{1} \Delta \tau \\
A_{7}=S_{r} \frac{2 \Delta \tau(\cos \beta \Delta Y-1)}{(\Delta Y)^{2}} \\
A_{8}=1+\frac{1}{S_{c}} \frac{2 \Delta \tau(\cos \beta \Delta Y-1)}{(\Delta Y)^{2}}-\Delta \tau K_{c}-U \frac{\Delta \tau\left(1-\mathrm{e}^{-i \alpha \Delta X}\right)}{\Delta X}-V \frac{\Delta \tau\left(\mathrm{e}^{i \beta \Delta Y}-1\right)}{\Delta Y}
\end{gathered}
$$

The Equations (31)-(33) can be designated in matrix-vector form are as follows:

$$
\left[\begin{array}{l}
F^{\prime} \\
G^{\prime} \\
H^{\prime}
\end{array}\right]=\left[\begin{array}{ccc}
A_{1} & A_{2} & A_{3} \\
A_{4} & A_{5} & A_{6} \\
0 & A_{7} & A_{8}
\end{array}\right]\left[\begin{array}{l}
F \\
G \\
H
\end{array}\right]
$$

Equation (42) is implied in $\eta^{\prime}=T^{*} \eta$ where $\eta^{\prime}=\left[\begin{array}{c}F^{\prime} \\ G^{\prime} \\ H^{\prime}\end{array}\right], \quad T^{*}=\left[\begin{array}{ccc}A_{1} & A_{2} & A_{3} \\ A_{4} & A_{5} & A_{6} \\ 0 & A_{7} & A_{8}\end{array}\right], \quad \eta=\left[\begin{array}{c}F \\ G \\ H\end{array}\right]$

To obtain the stability criterion, it is sufficient to find out eigenvalues of the amplification matrix $T^{*}$ but this study is very difficult since all the elements of $T^{*}$ are different. So the time-step $\Delta \tau$ is considered to be very small and tends to zero. Under this assumption we have $A_{2} \rightarrow 0, A_{3} \rightarrow 0, A_{4} \rightarrow 0, A_{6} \rightarrow 0, A_{7} \rightarrow 0$, and the amplification matrix takes the following form

$$
T^{*}=\left[\begin{array}{ccc}
A_{1} & 0 & 0 \\
0 & A_{5} & 0 \\
0 & 0 & A_{8}
\end{array}\right]
$$

The eigenvalues of the amplification matrix $T^{*}$ are obtained as $\lambda_{1}=A_{1}$, $\lambda_{2}=A_{5}, \lambda_{3}=A_{8}$. For stability test each of the eigenvalues must not exceed unity in modulus. Under this consideration, the stability conditions are as follows:

$$
\left|A_{1}\right| \leq 1,\left|A_{5}\right| \leq 1,\left|A_{8}\right| \leq 1
$$

Let we choose,

$$
a_{1}=\Delta \tau, b_{1}=U \frac{\Delta \tau}{\Delta X}, c_{1}=|-V| \frac{\Delta \tau}{\Delta X} \text { and } d_{1}=2 \frac{\Delta \tau}{(\Delta Y)^{2}}
$$

Also the coefficients of $a_{1}, b_{1}$ and $c_{1}$ are all real and nonnegative. Now we con- 
sider that $U$ is everywhere nonnegative and $V$ is everywhere non-positive. We can demonstrate that the maximum modulus of $A_{1}, A_{5}$ and $A_{8}$ occurs when $\alpha \Delta X=m \pi$ and $\beta \Delta Y=n \pi$, where $m$ and $n$ are integers. The Expressions (45) can occur when $m$ and $n$ both are odd integers. Thus the Expression (45) can be written into the followings form

$$
\begin{gathered}
A_{1}=1-2\left[d_{1}+a_{1}\left(M+\frac{1}{D a}\right)+b_{1}+c_{1}+\frac{F_{s}}{D a} U\right] \\
A_{5}=1-2\left[d_{1} \frac{1}{P_{r}}\left(1+\frac{4}{3} R\right)-\frac{a_{1}}{2}\left(Q-U^{2} E_{c} M \varepsilon\right)+b_{1}+c_{1}\right] \\
A_{8}=1-2\left[d_{1} \frac{1}{S_{c}}+b_{1}+c_{1}+\frac{a_{1}}{2} K_{c}\right]
\end{gathered}
$$

The most negative allowable values of $A_{1}, A_{5}$ and $A_{8}$ are -1 . Then the stability conditions for the present problem are as furnished, upon simplification of Equations (45)-(47)

$$
\begin{gathered}
U \frac{\Delta \tau}{\Delta X}+|V| \frac{\Delta \tau}{\Delta Y}+\Delta \tau\left(M+\frac{1}{D a}\right)+\Delta \tau \frac{F_{s}}{D a} U \leq 1 \\
U \frac{\Delta \tau}{\Delta X}+|V| \frac{\Delta \tau}{\Delta Y}+\frac{2 \Delta \tau}{(\Delta Y)^{2}} \frac{1}{P_{r}}\left(1+\frac{4}{3} R\right)-\frac{\Delta \tau Q}{2}+\frac{\Delta \tau U^{2} E_{c} M \varepsilon}{2} \leq 1 \\
U \frac{\Delta \tau}{\Delta X}+|V| \frac{\Delta \tau}{\Delta Y}+\frac{2 \Delta \tau}{(\Delta Y)^{2}} \frac{1}{S_{c}}+\frac{\Delta \tau K_{c}}{2} \leq 1
\end{gathered}
$$

Since from the initial conditions $U=V=0$ at $\tau=0$, the Equations (49) and (50) provide $P_{r} \geq 0.05$ and $S_{c} \geq 0.03$ respectively.

Hence the convergence tests of the present problem are $P_{r} \geq 0.05$ and $S_{c} \geq 0.03$.

\section{Results and Discussion}

In order to get into the physical understanding of the problem, non-dimensional velocity, temperature, concentration, skin friction, Nusselt number and Sherwood number are computed for various values of acceleration parameter $(a)$, inclination angle $(\alpha)$, Grashof number $\left(G_{I}\right)$, Mass Grashof number $\left(G_{m}\right)$, Magnetic parameter $(M)$, electrical conductivity parameter $(\varepsilon)$ Darcy number $\left(D_{a}\right)$, Forchheimer number $\left(F_{s}\right)$, Prandtl number $\left(P_{r}\right)$, radiation parameter $(R)$, Heat generation parameter $(Q)$, radiation absorption parameter $\left(Q_{1}\right)$, Dufour number $\left(\mathrm{D}_{\mathrm{u}}\right)$, Eckert number $\left(E_{c}\right)$, Schmidt number $\left(S_{c}\right)$, Soret number $\left(S_{r}\right)$, Chemical reaction parameter $\left(K_{c}\right)$ and the order of chemical reaction (I). Grashof number governs the fluid flow, in the case of free convection flow. The significance of the Grashof number is that it designates the ratio between the buoyancy forces to the viscous forces. The value of Grashof number is assumed positive to represent the cooling of the plate. Prandtl number $\left(P_{r}\right)$ is a measure of the relative significance of momentum and thermal boundary layers. Prandtl number $(P r)$ is approximately constant, for most gases over a wide range of temperature and 
pressure. The values of the Prandtl number $P_{r}$ are considered for air $\left(P_{r}=0.71\right)$, the electrolytic solution $\left(P_{r}=1.00\right)$, sulfur dioxide $\left(P_{r}=4.24\right)$, water $\left(P_{r}=7.00\right)$ and water at $4^{\circ} \mathrm{C}\left(P_{r}=11.62\right)$. Furthermore, it can be used to measure the thermal conductivity of gases at high temperatures, where it is very difficult to measure experimentally due to the formation of convection currents. In heat transfer problems, the Prandtl number $\left(P_{r}\right)$ has significant effect on the relative thickness of the momentum and thermal boundary layers. The heat diffuses more rapidly compared to the velocity, in the presence of low Prandtl number $\left(P_{r}\right)$. The values of the Schmidt number are taken to designate the presence of species by water vapor $\left(S_{c}=0.66\right)$, ammonia $\left(S_{c}=0.78\right)$ and carbon dioxide $\left(S_{c}=\right.$ 0.95). Physically, the Schmidt number relates to the relative thickness of the hydrodynamic boundary layer and mass transfer boundary layer. In order to obtain the accuracy of the numerical results, the following values of default parameter are chosen as: $G_{r}=10, G_{m}=5, D_{a}=1, F_{s}=0.1, M=1, \varepsilon=0.50, P_{r}=0.71, R=0.5$, $D_{u}=0.03, Q=0.1, Q_{1}=1, E_{c}=0.01, S_{c}=0.66, S_{r}=1.6, K_{c}=0.5, \tau=0.4, a=0.5$, $\alpha=\pi / 3$ and $I=2$. All graphs therefore correspond to these unless specifically indicated on the appropriate graph. The numerical solutions for the fluid velocity, temperature, concentration, skin-friction, Nusselt number and Sherwood number are presented graphically in Figures 2-24.

Figure 2 delineates the effect of angle of inclination $\varphi$ on fluid flow. Velocity decreases with the increasing values of inclination angle because the magnitude of the buoyancy force decreases with an increase in the inclination angle. Pattnaik et al. [27] are computed the same result.

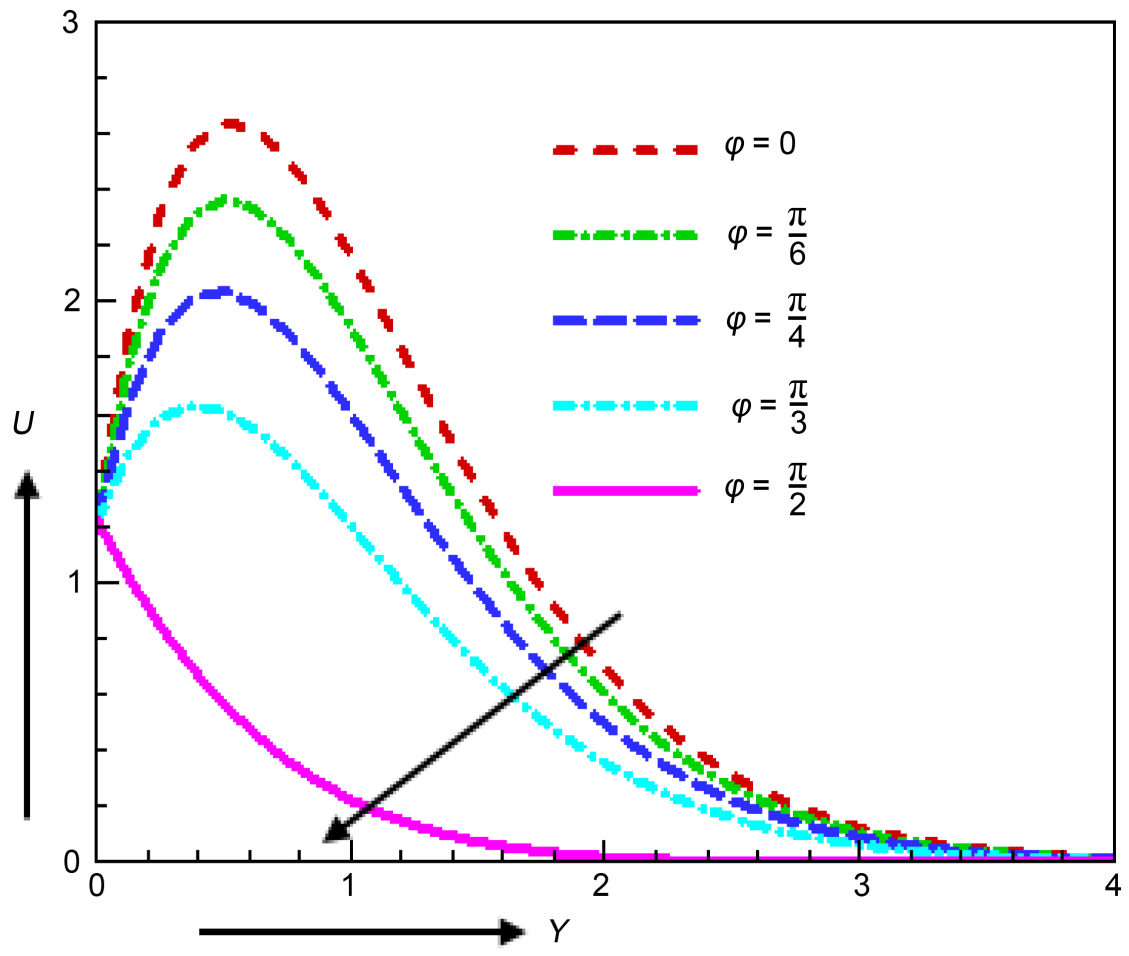

Figure 2. Velocity profile for various values of $\varphi$. 


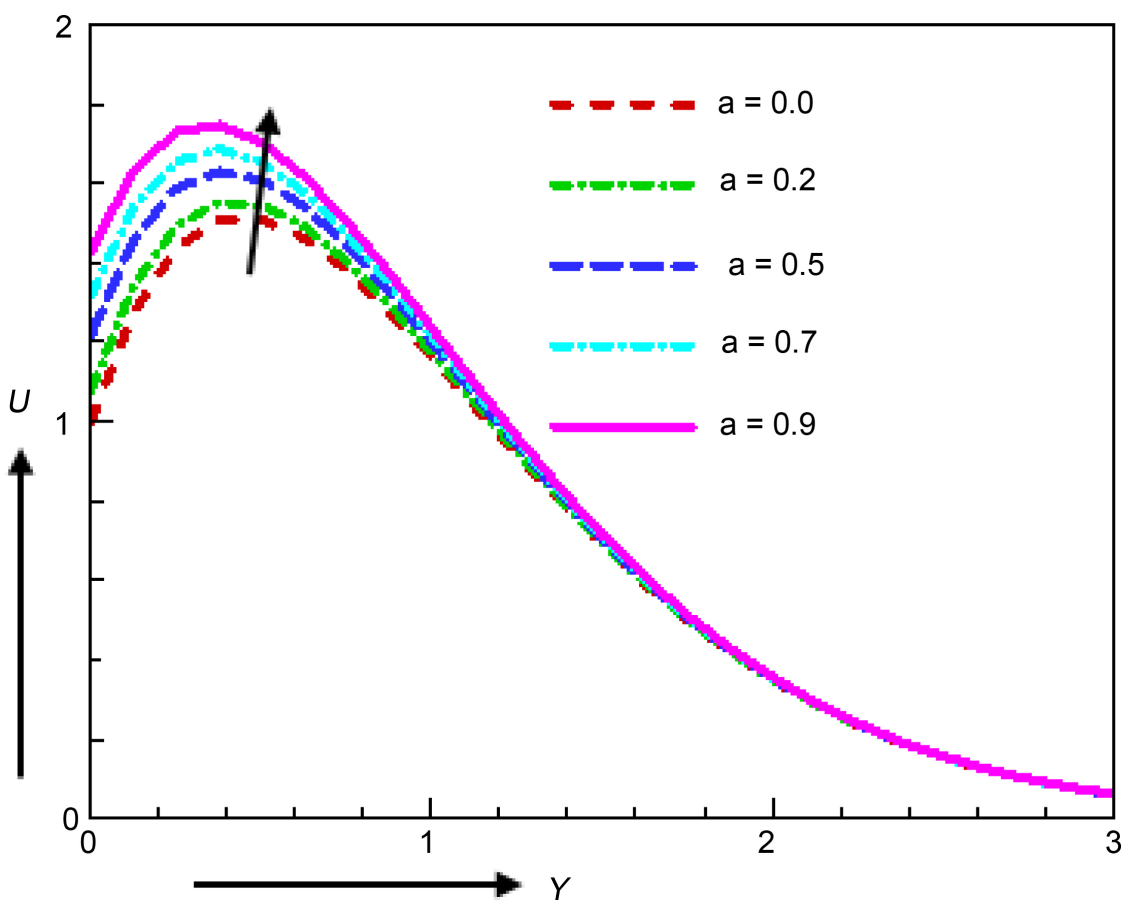

Figure 3. Velocity profile for various values of $a$.

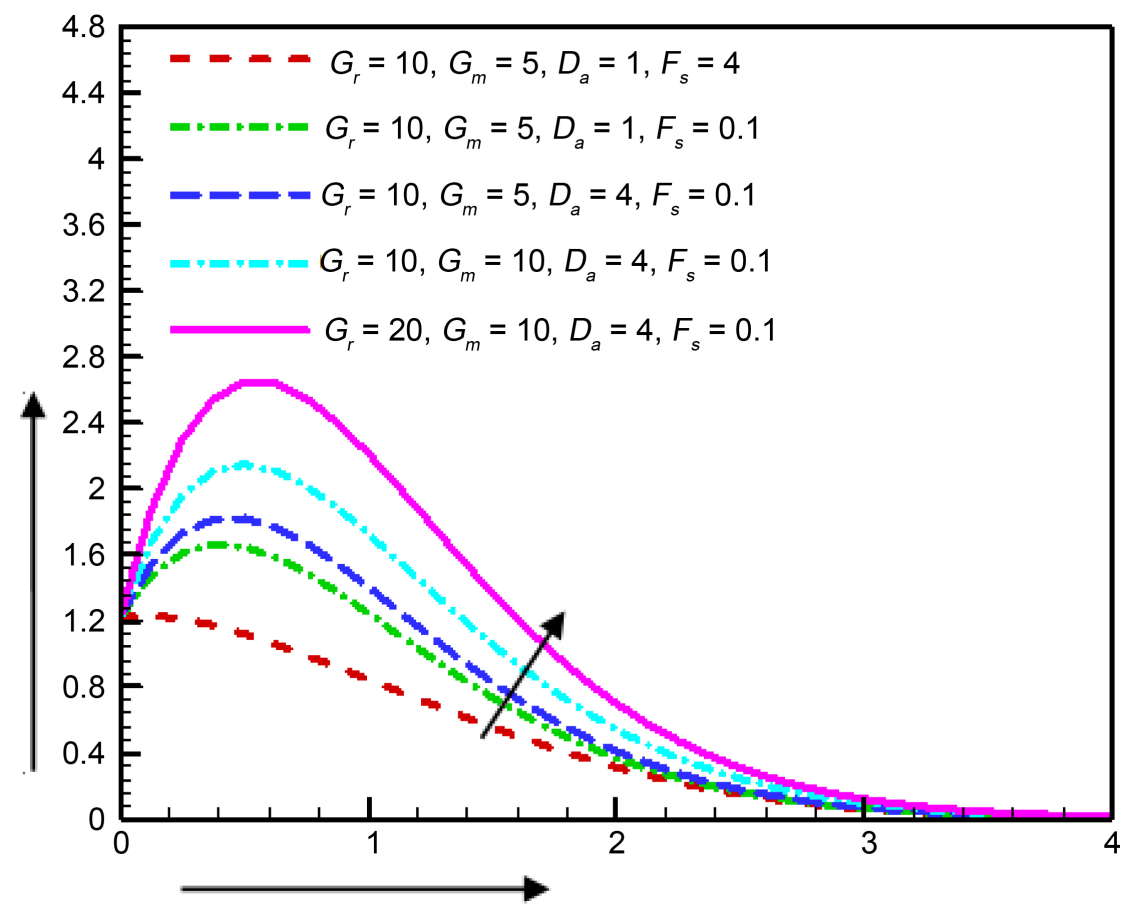

Figure 4. Velocity profile for various values of $G_{r}, G_{m}, D_{a}$ and $F_{s}$.

Figure 3 demonstrates the impact of acceleration parameter $a$ on the velocity field. It is evident that the velocity increases near the plate and remains same far away from the plate with the increase of acceleration parameter $a$. The same result has already been deliberated by Pattnaik et al. [27]. 


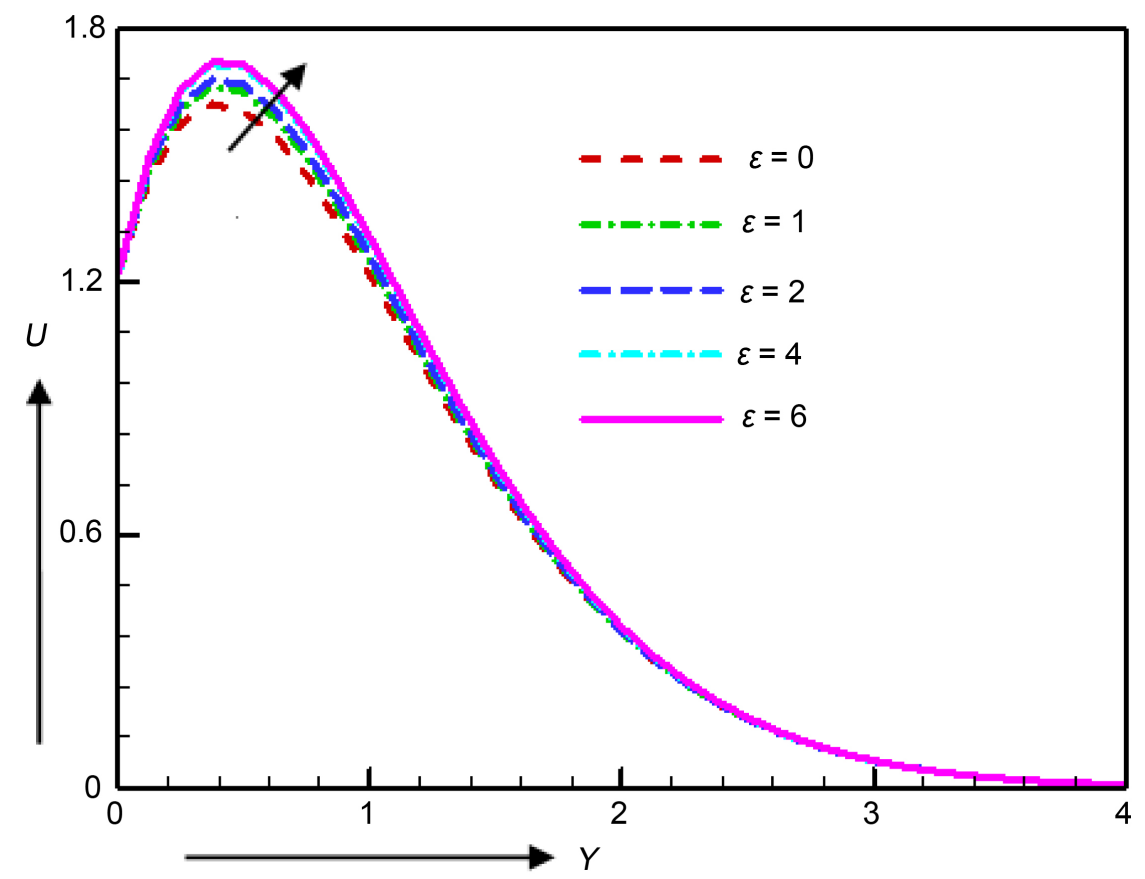

Figure 5. Velocity profile for various values of $\varepsilon$.

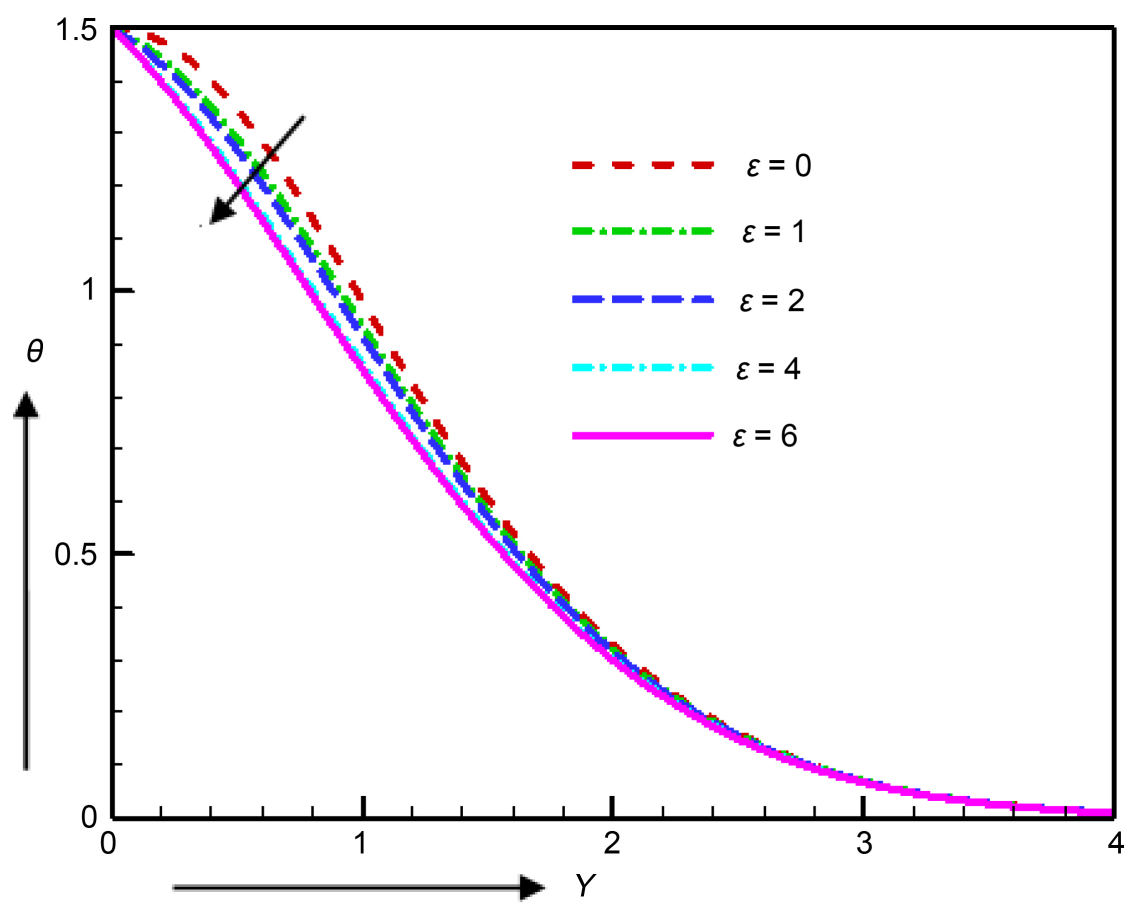

Figure 6. Temperature profile for various values of $\varepsilon$.

The effect of a velocity profile for different values of Grashof number $G_{r}$, mass Grashof number $G_{m}$, Darcy number $D_{a}$ and Forchheimer number $F_{s}$ are depicted in Figure 4. The Grashof number approximates the ratio of the thermal buoyancy force to the viscous hydrodynamic force acting on a fluid. The negative 


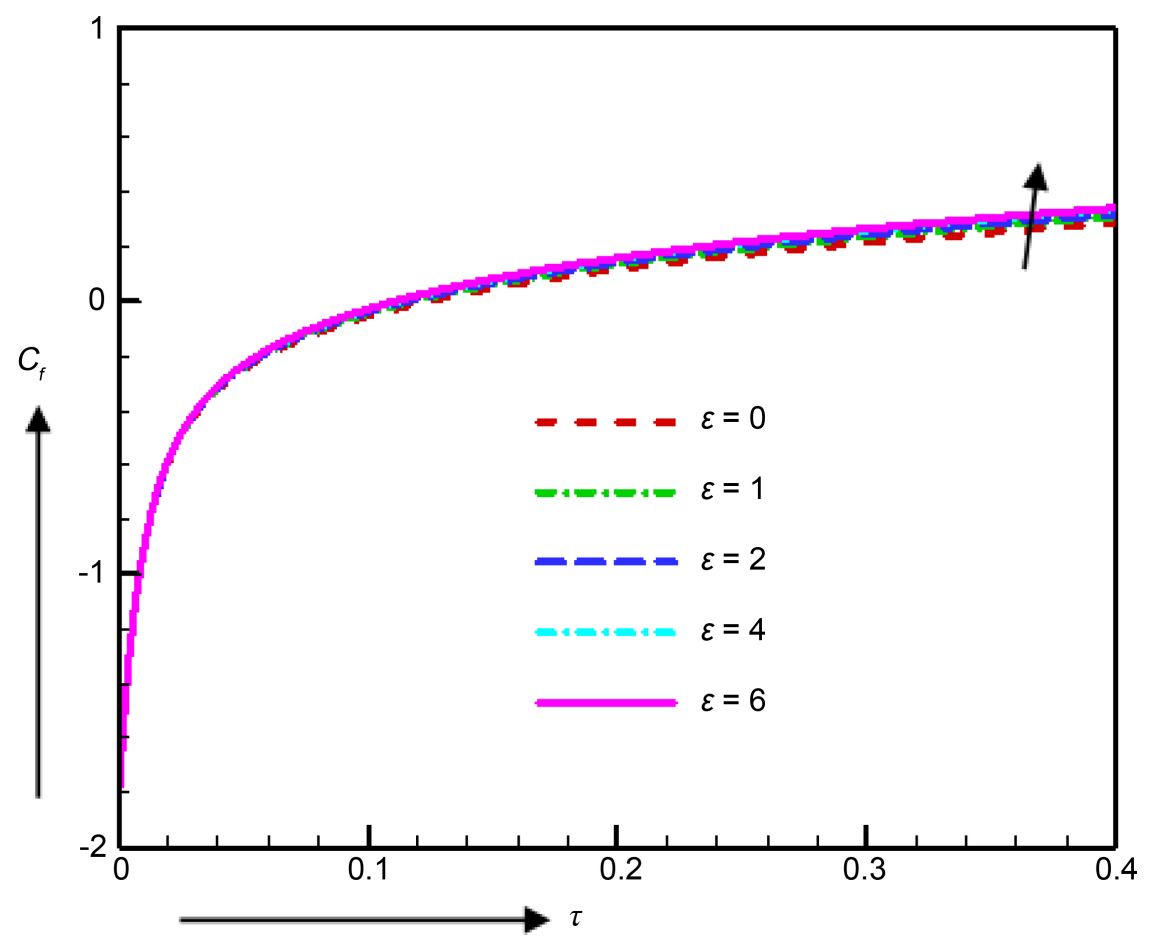

Figure 7. Skin friction for various values of $\varepsilon$.

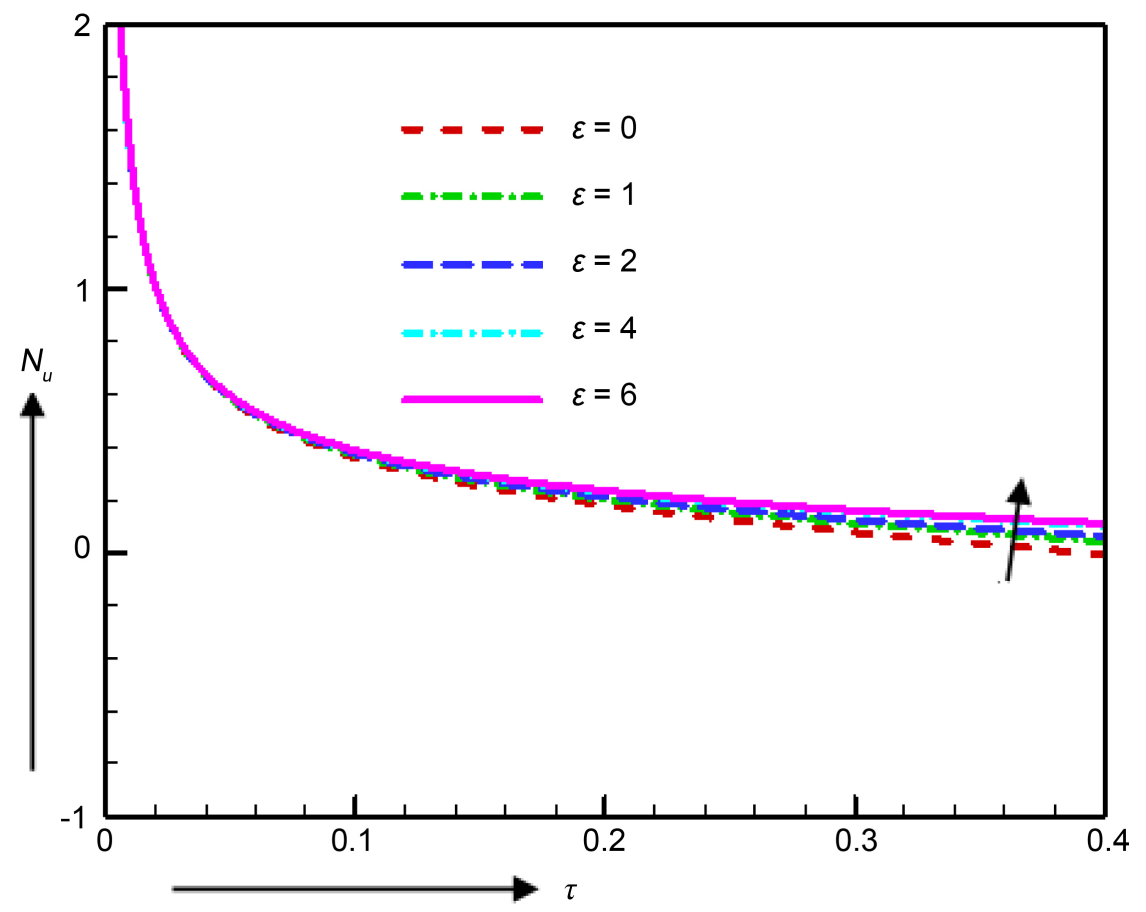

Figure 8. Nusselt number for various values of $\varepsilon$.

value of Grashof number means that the plate is heating and the positive value represents that the plate is cooling. The null value of Grashof number represents the absence of free convection current. The mass Grashof number $G_{m}$ designates 


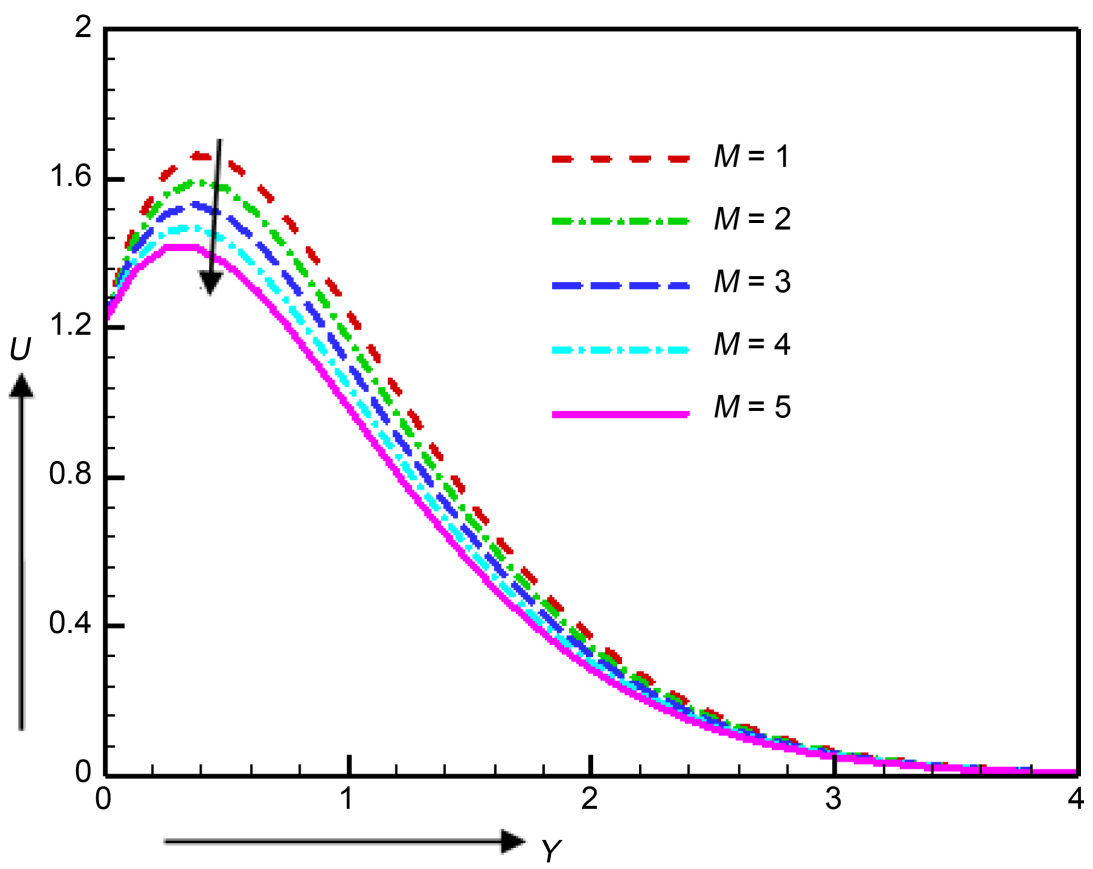

Figure 9. Velocity profile for various values of $M$.

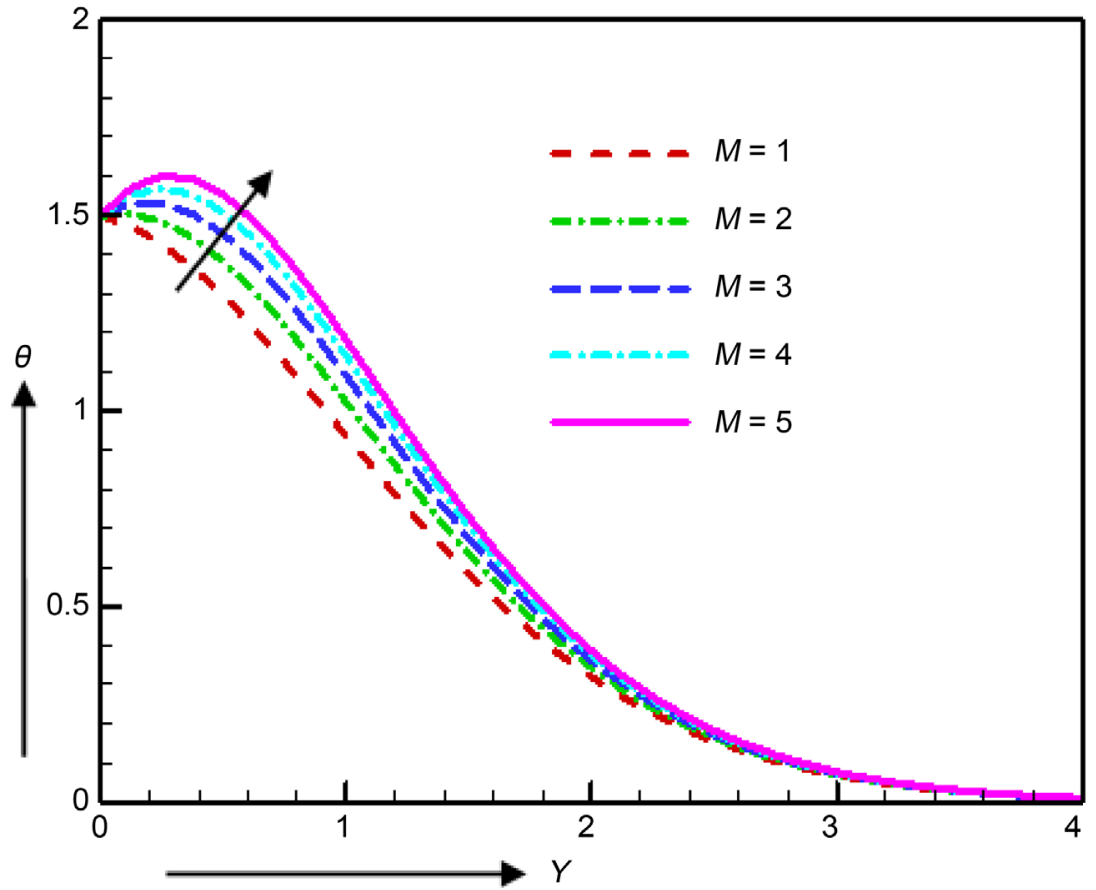

Figure 10. Temperature profile for various values of $M$.

the ratio of the concentration buoyancy force to the viscous hydrodynamic force. The porosity of the medium increases due to the presence of Darcy number $D_{a}$, thus fluid flows rapidly. Forchheimer number $F_{s}$ represents the inertial drag, hence the fluid velocity decreases with the increase in the Forchheimer number. As expected, it is noticed that there is a rise in the velocity owing to the increase 


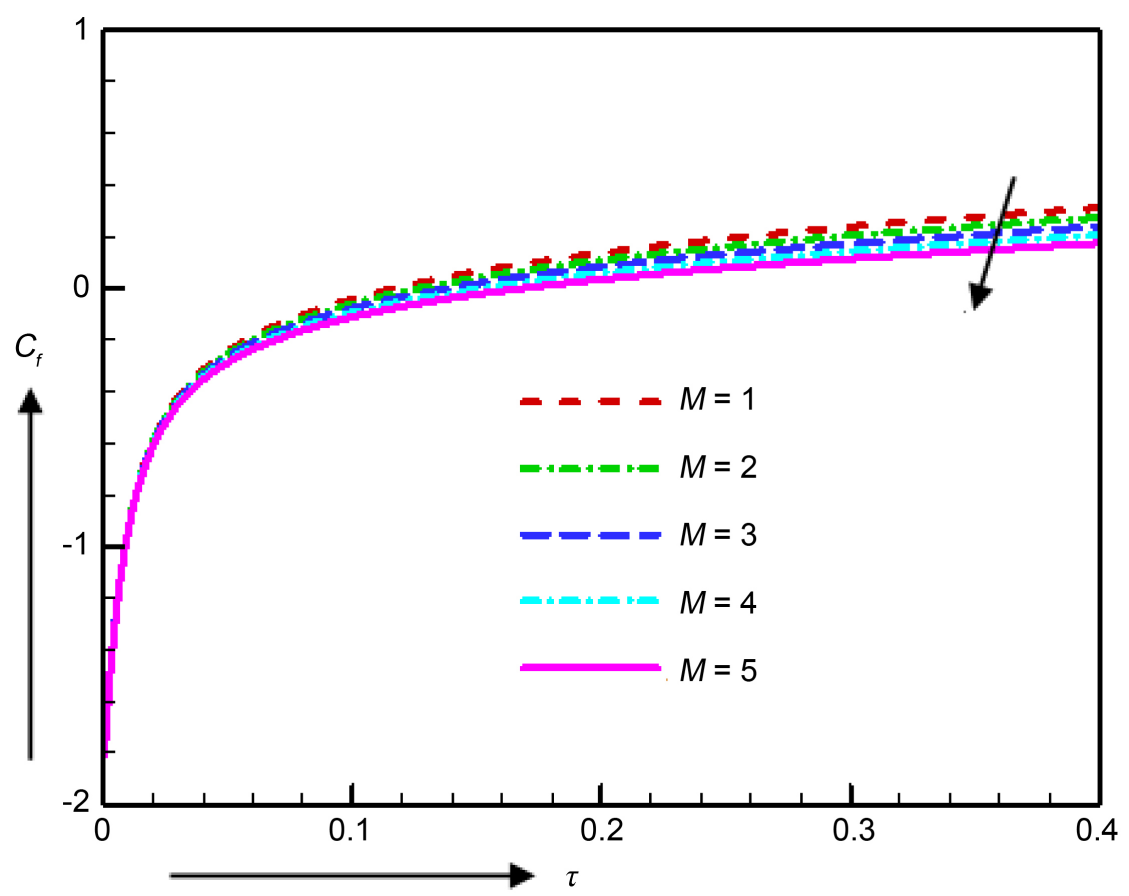

Figure 11. Skin friction for various values of $M$.

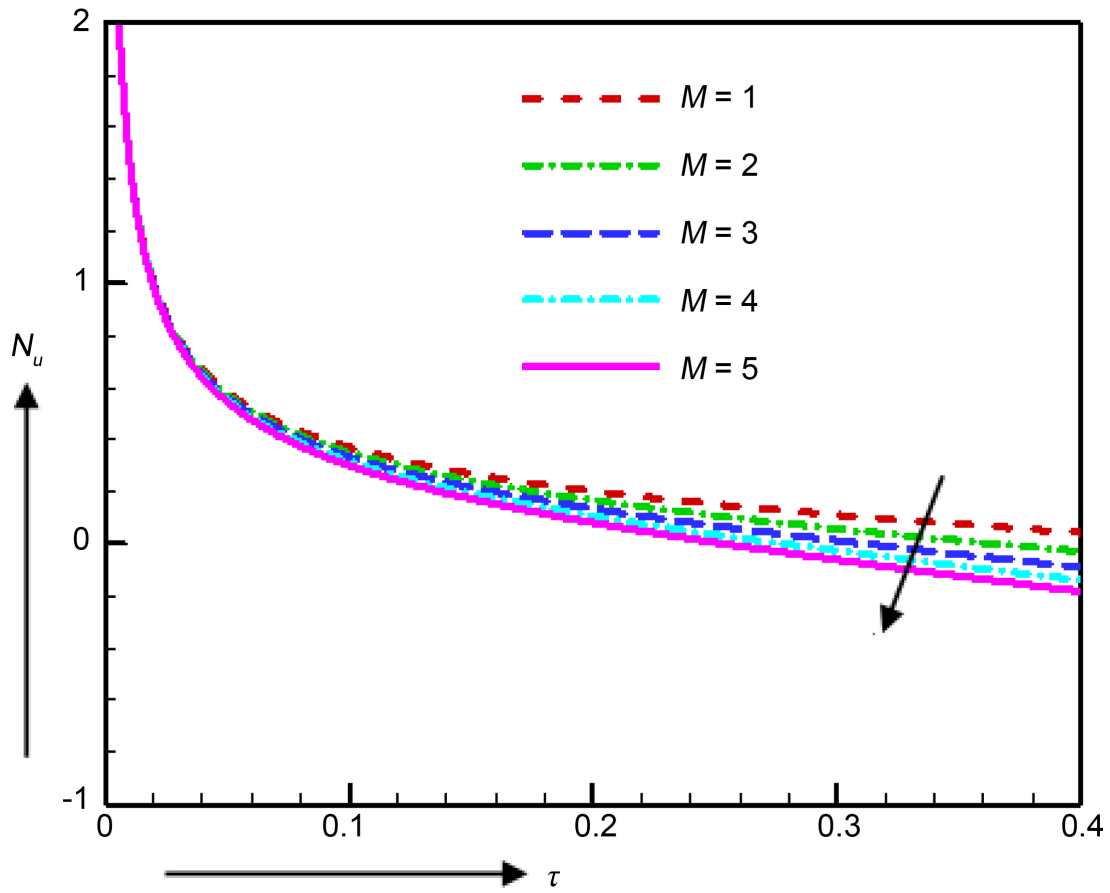

Figure 12. Nusselt number for various values of $M$.

of thermal buoyancy force, species buoyancy force and Darcy number while it decreases with the increase of Forchheimer number.

The importance of electrical conductivity parameter $\varepsilon$ on velocity, temperature, skin friction and Nusselt number are revealed in Figures 5-8. Velocity as 


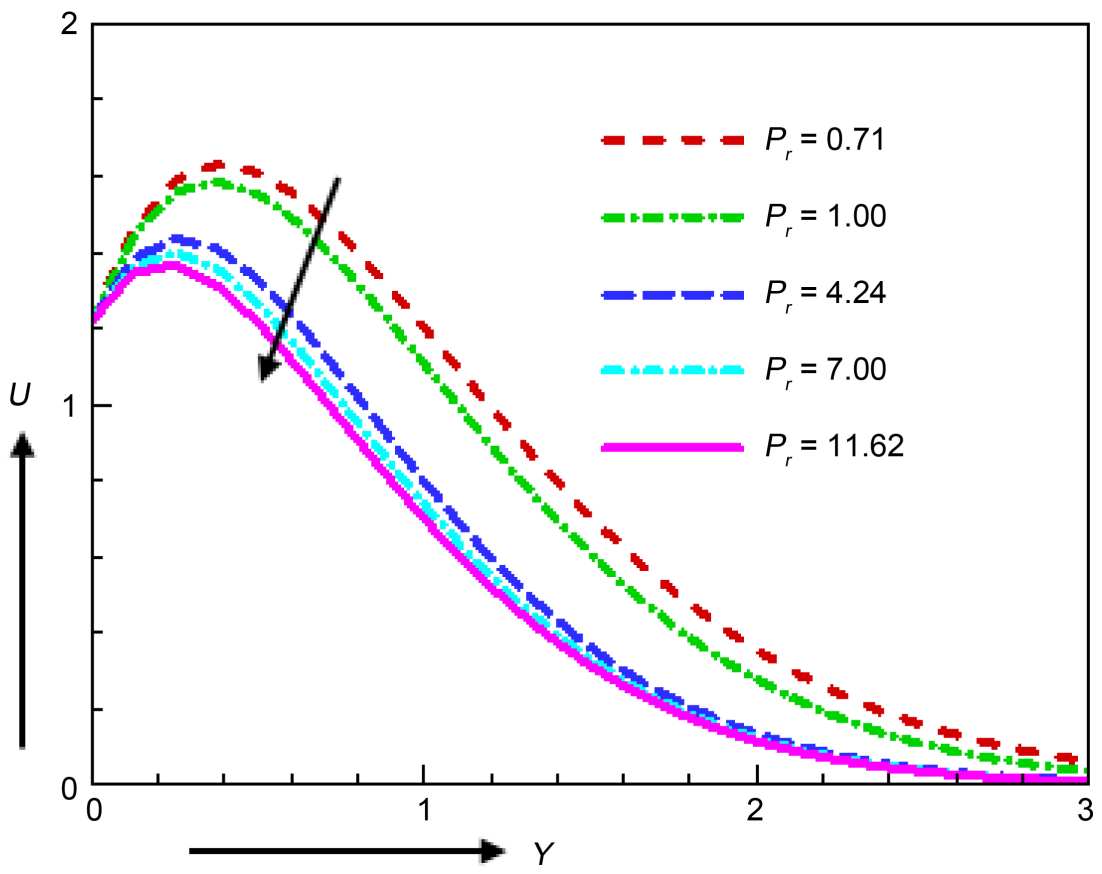

Figure 13. Velocity profile for various values of $P_{r}$

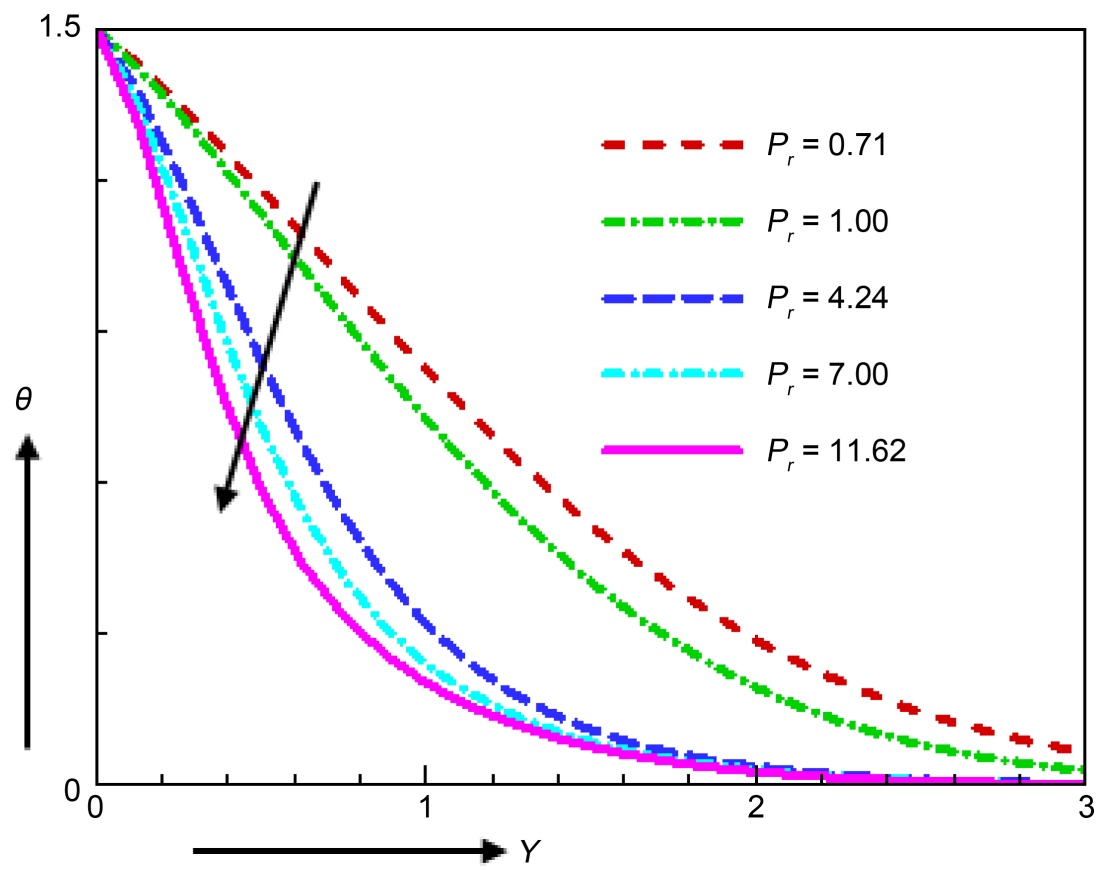

Figure 14. Temperature profile for various values of $P_{r}$

well as local skin friction increase due to the increase of electrical conductivity parameter $\mathcal{E}$. A similar result has been noticed by Shrama and Singh [28]. At $Y=$ 0.375 , the values of velocities are computed numerically as $1.62184,1.6639,1.68$, 1.71604 and 1.72238 for electrical conductivity parameter $\varepsilon=0,1,2,4$ and 6 respectively. Here it is noticed that the maximum velocity increases by $4.206 \%$ as 


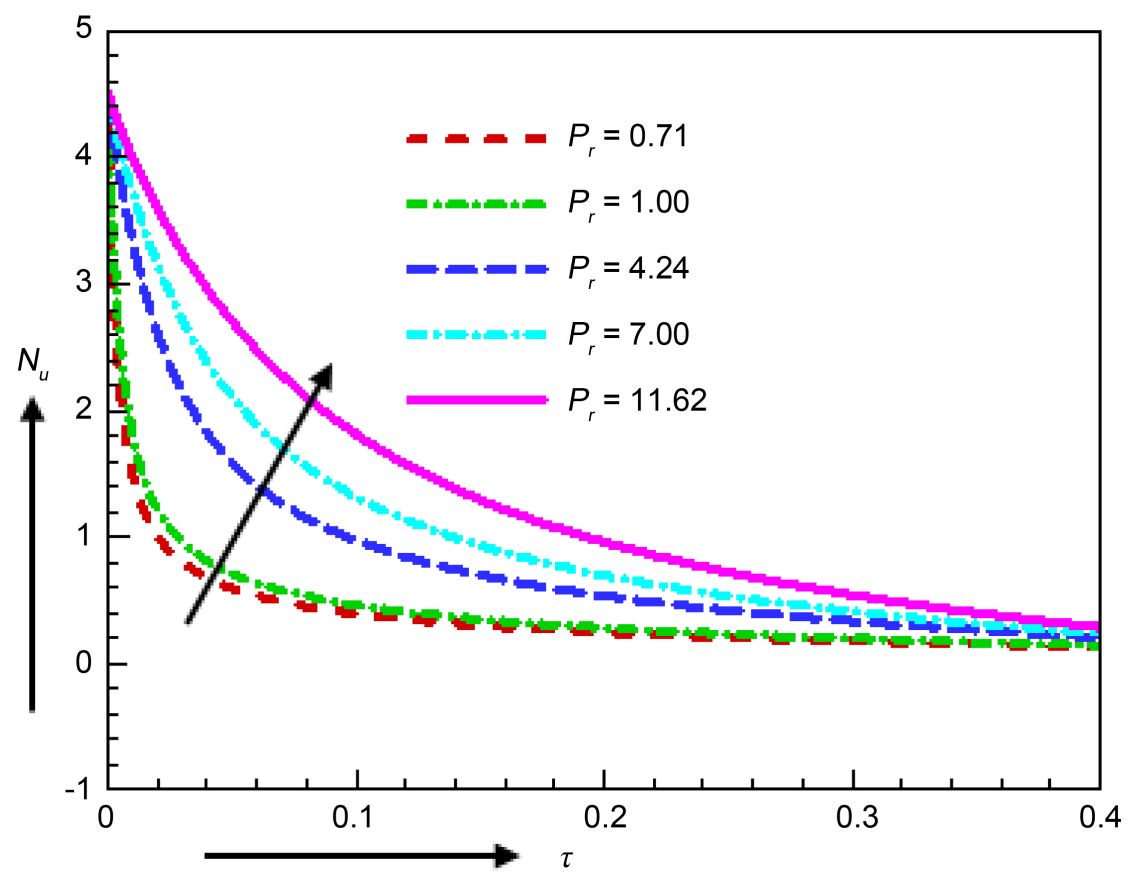

Figure 15. Nusselt number for various values of $P_{r^{*}}$

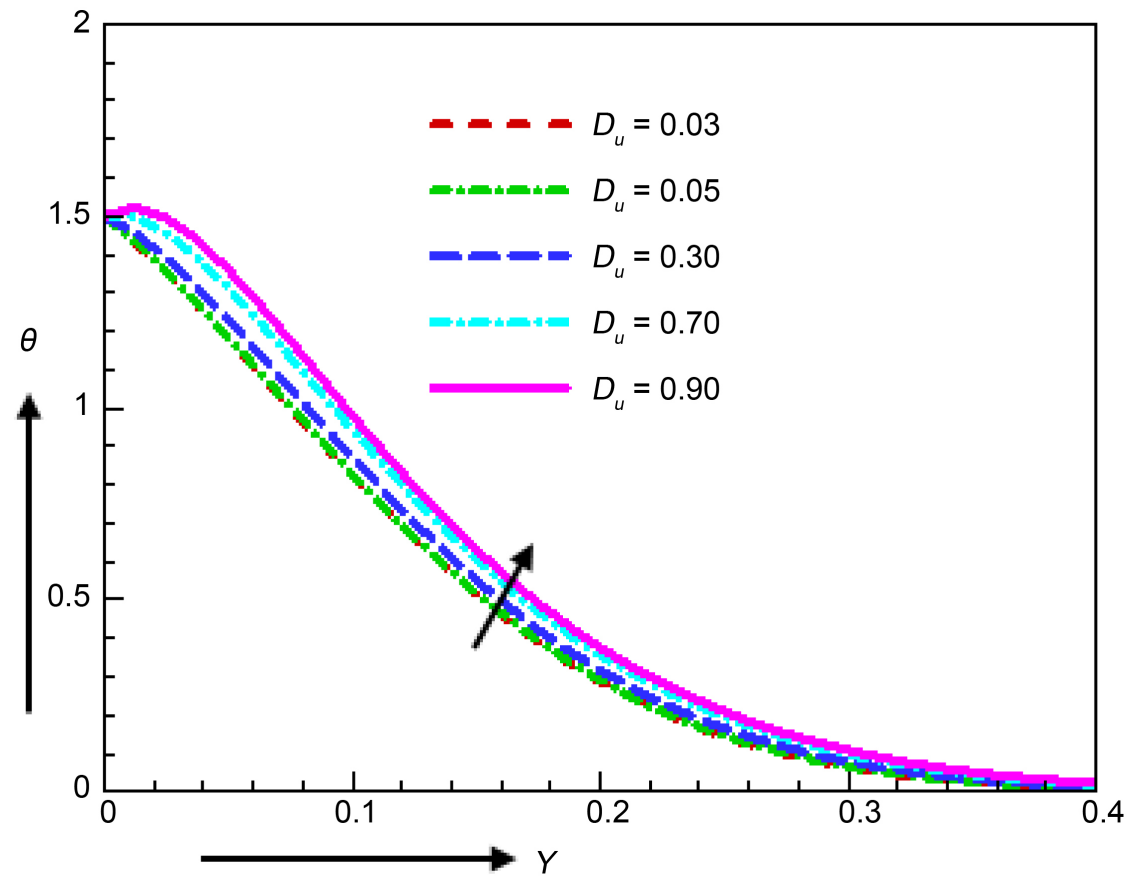

Figure 16. Temperature profile for various values of $D_{u}$.

the electrical conductivity parameter $\varepsilon$ change from 0 to 1 . The values of temperature are given as $1.34834,1.29251,1.26982,1.216$ and 1.20606 for electrical conductivity parameter $\varepsilon=0,1,2,4$ and 6 respectively which take place at same position $Y=0.5$ and the maximum temperature increases by $5.584 \%$ as the change of $\varepsilon=0$ to $\varepsilon=1$. Electrical conductivity parameter $\varepsilon$ generates the 


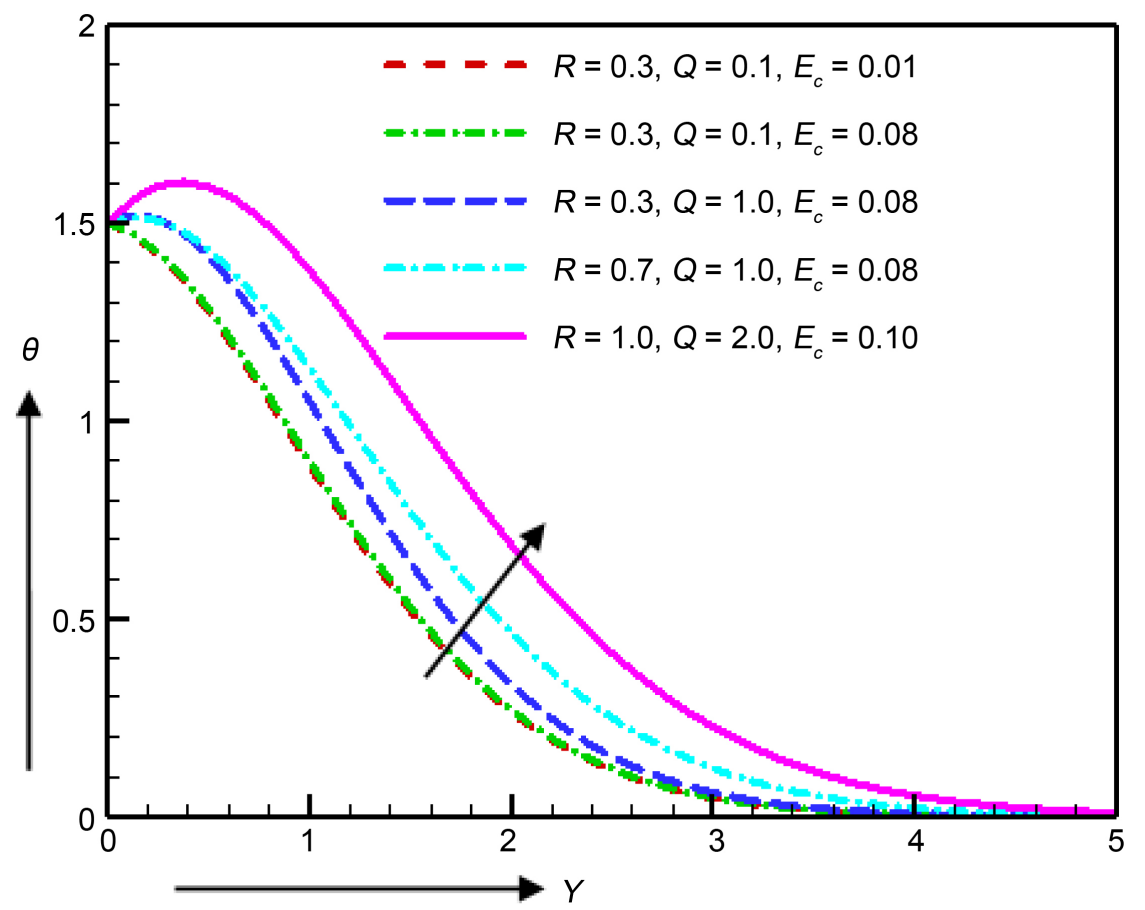

Figure 17. Temperature profile for various values of $R, Q$ and $E_{c}$

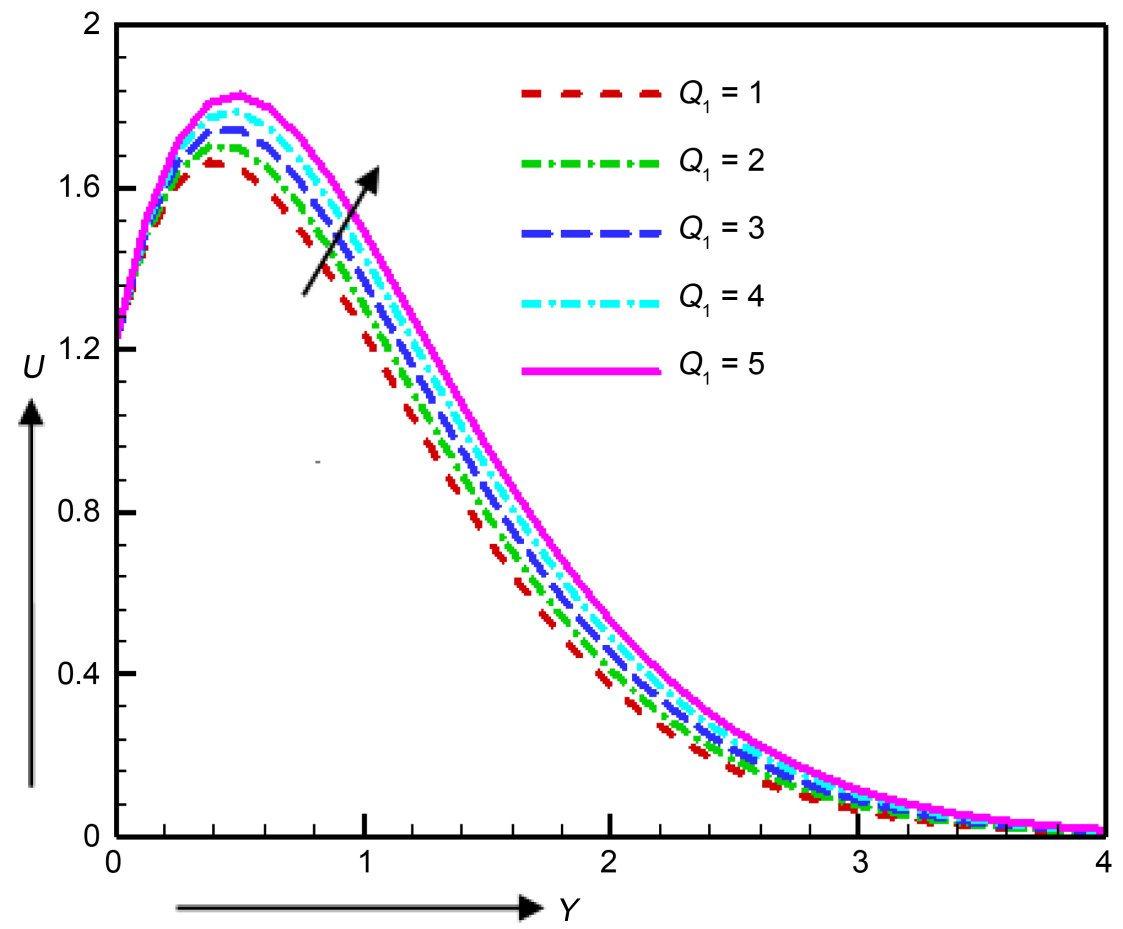

Figure 18. Velocity profile for various values of $Q_{1}$.

buoyancy force that leads to rising the fluid motion and skin friction. In the presence of high-speed MHD, electrical conductivity parameter decreases the thermal buoyancy force which decreases the temperature corresponds to the rise 


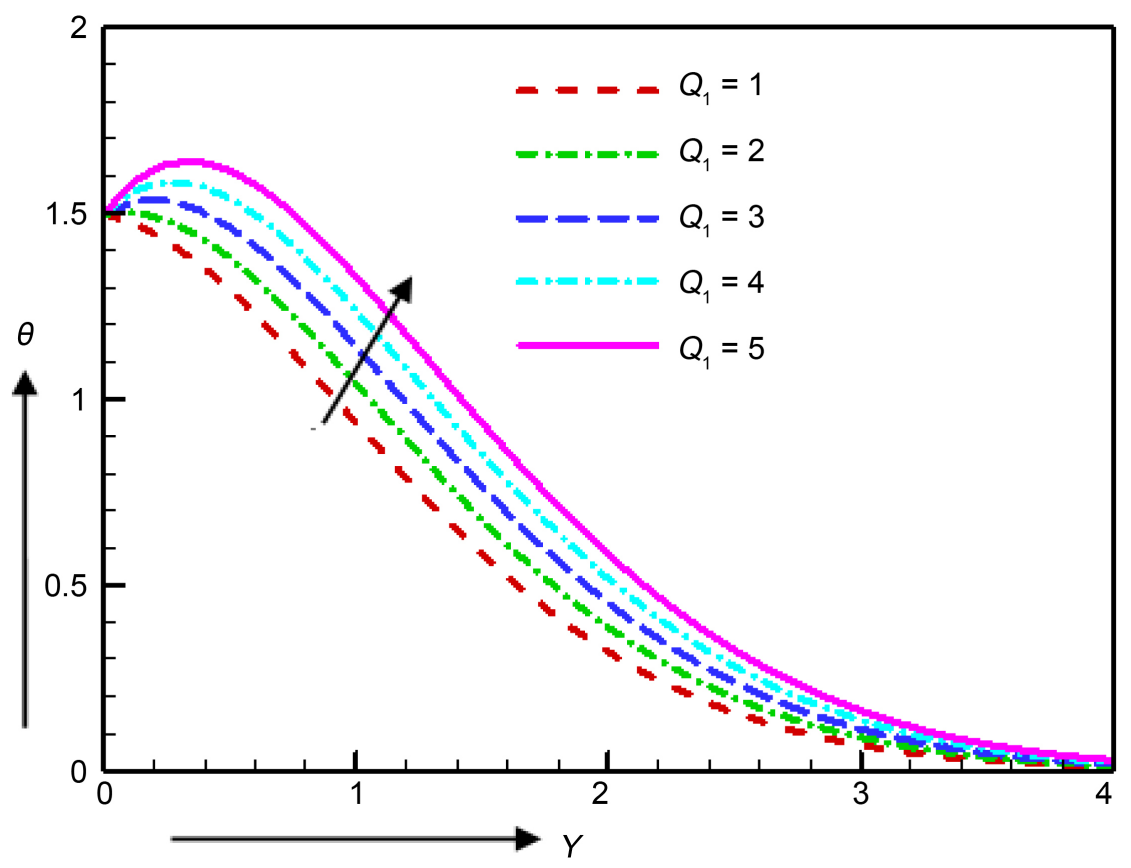

Figure 19. Temperature profile for various values of $Q_{1}$.

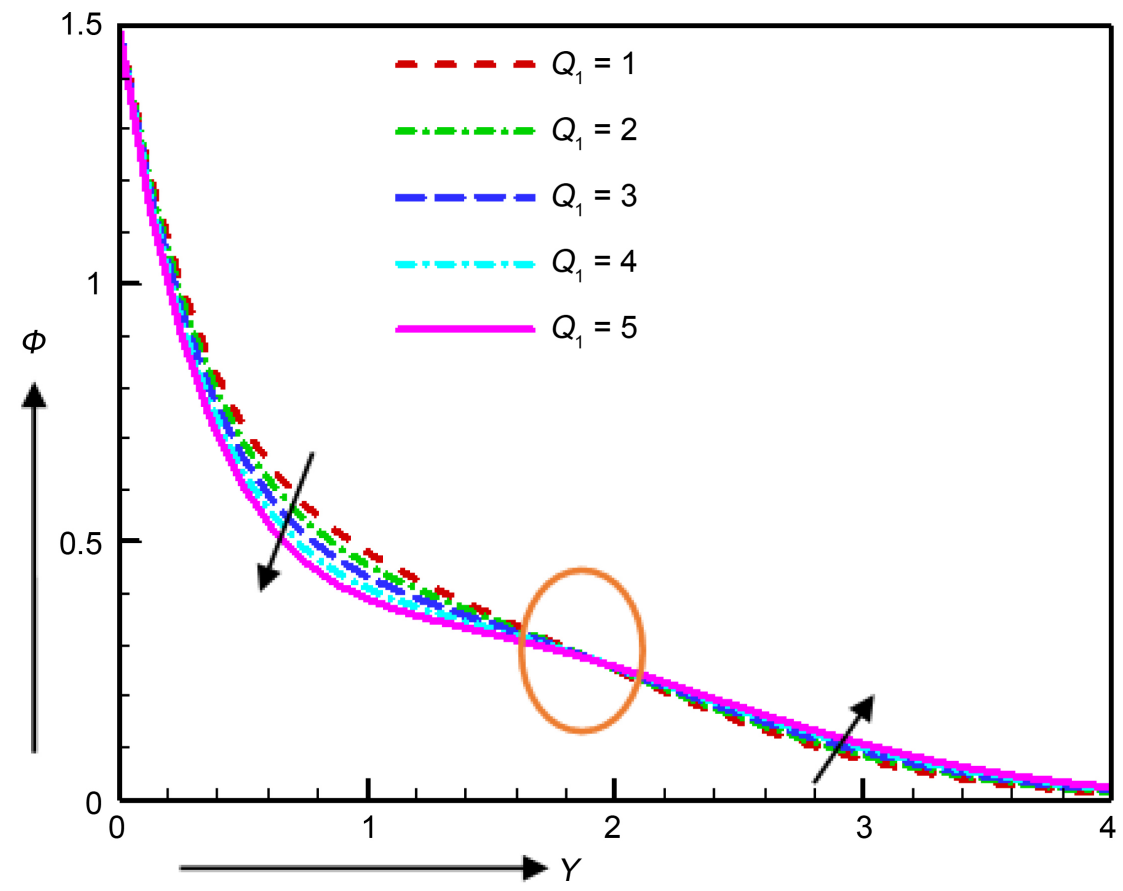

Figure 20. Concentration profile for various values of $Q_{1}$.

in the rate of heat transfer.

The high speed MHD effects on velocity, temperature, skin friction and Nusselt number are shown in Figures 9-12. Transverse magnetic field generates a resistive force which takes the reduction in the fluid velocity. Thus the velocity of the flow field decreases in the presence of magnetic field. Velocity decreases 


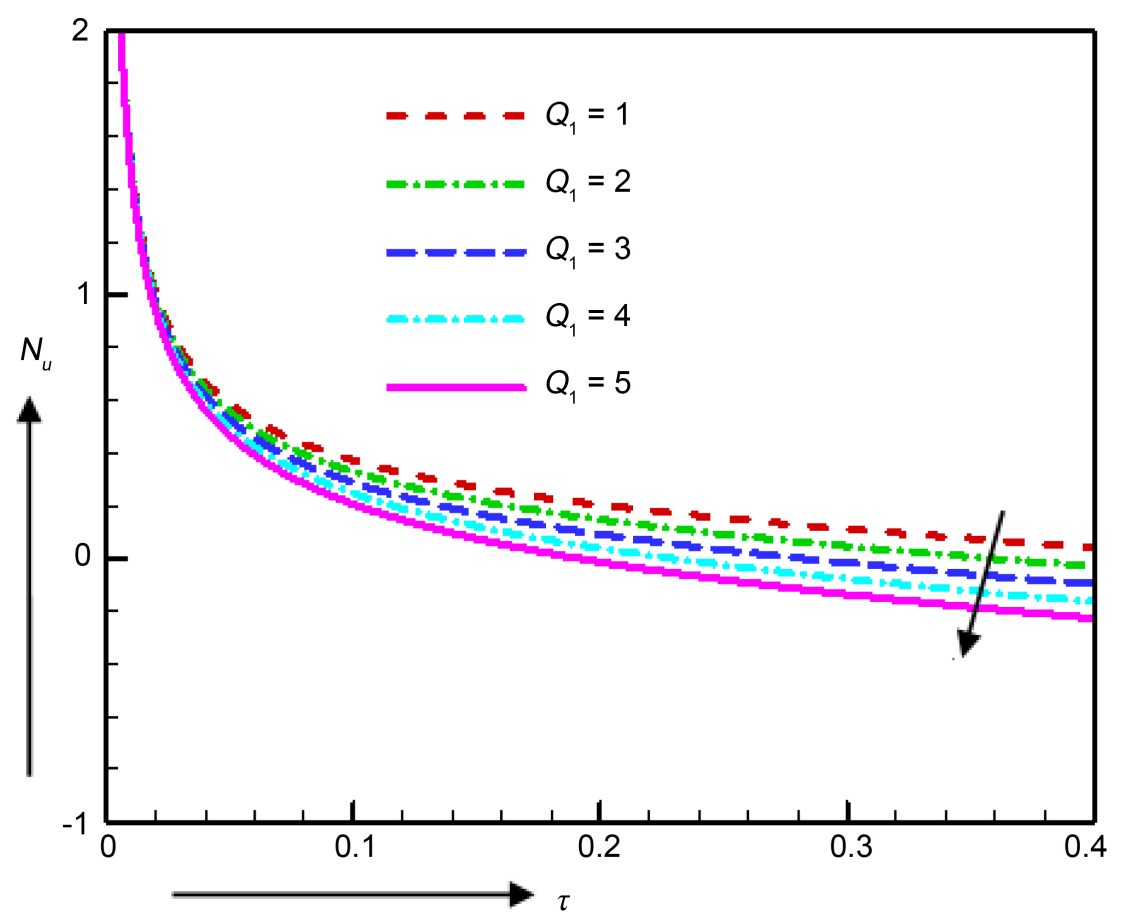

Figure 21. Nusselt number for various values of $Q_{1}$.

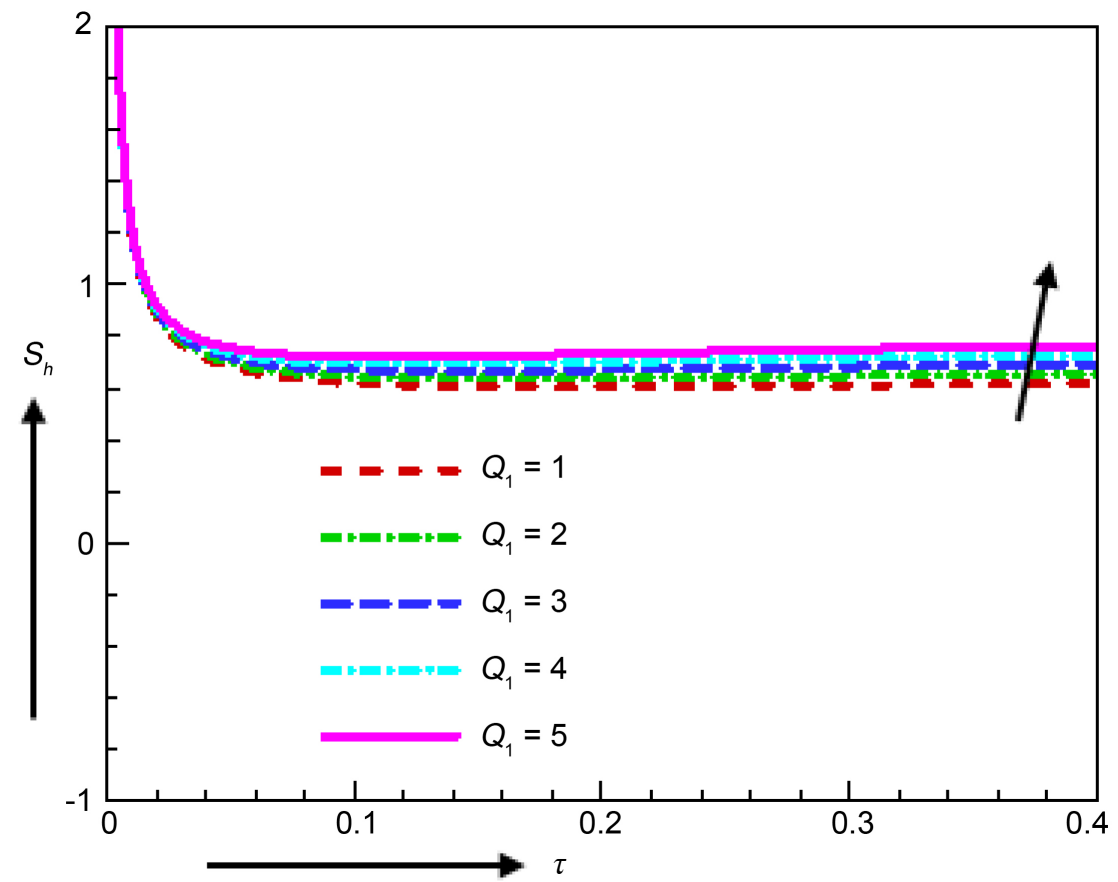

Figure 22. Sherwood number for various values of $Q_{1}$.

$6.904 \%, 6.413 \%, 5.987 \%$ and $5.611 \%$ for the change of the magnetic parameter $M$ as 1 to 2,2 to 3,3 to 4 and 4 to 5 respectively at $Y=0.375$. With increasing values of magnetic parameter, thermal buoyancy force increases in the presence of high-speed MHD which therefore enhances the temperature and rate of heat 


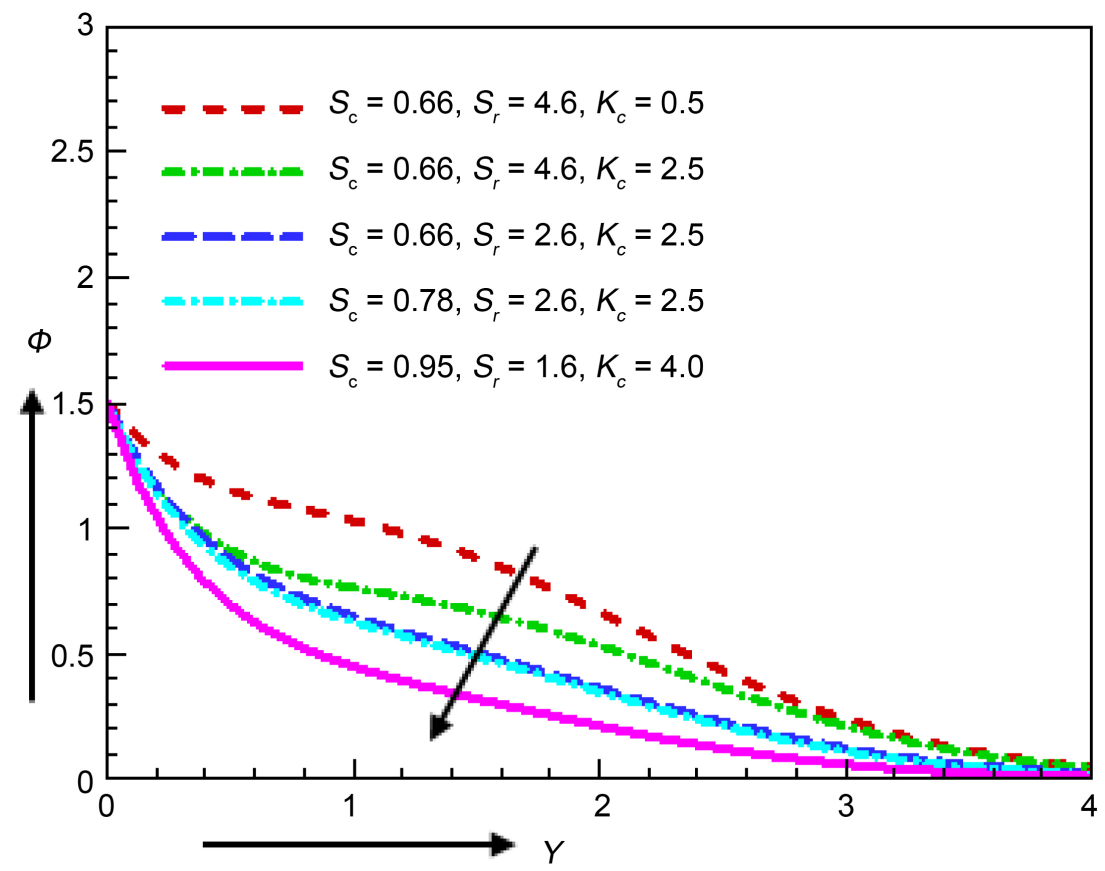

Figure 23. Concentration profile for various values of $S_{\mathcal{c}}, S_{r}$ and $K_{c}$

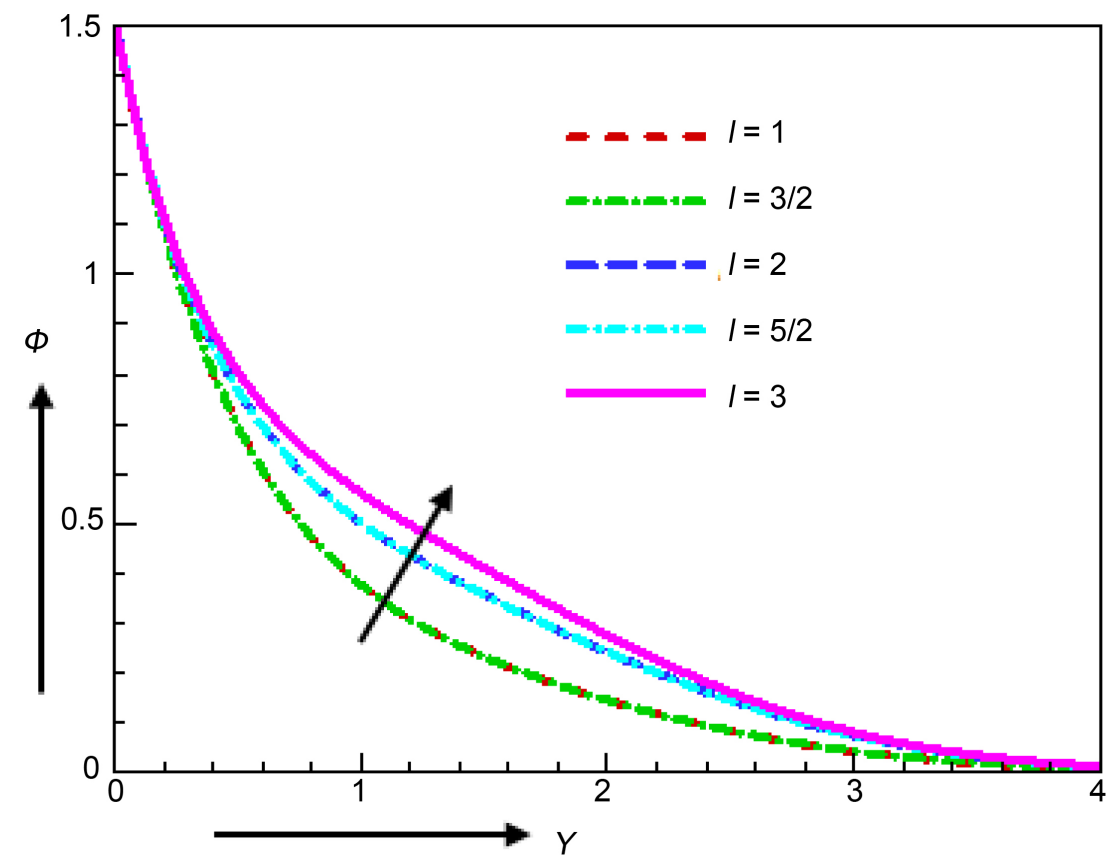

Figure 24. Concentration profile for various values of 1 .

transfer. The same type of result for velocity and temperature has already been illustrated by Desale and Pradhan [15] and Haque and Sarder [17]. At $Y=0.5$, the values of temperature are filed as 1.29251, 1.59486, 1.53073, 1.47086 and 1.41475 for the difference values of magnetic parameter $M$ as 1, 2, 3, 4 and 5 respectively. Here we observed that the maximum temperature increases by $8.855 \%$ as for the change of the magnetic parameter $M$ from 1 to 2 at the same 
point $Y=0.5$.

Figures 13-15 exhibit the effect of Prandtl number on velocity, temperature and Nusselt number. It is observed that higher values of Prandtl number decrease the velocity and temperature at all points of the flow domain. Due to the increase in Prandtl number Pr leads to the downfall of fluid temperature that means the temperature difference between the fluid and the plate rises and the associated heat transfer rate increases.

The influence of Dufour number $D_{u}$ number on temperature profile is delineated in Figure 16. The energy flux generated by concentration gradient represents the Dufour Effect. From Figure 16, it is clearly seen that temperature increase owing to the increase of Dufour number $D_{u}$.

The impacts of radiation parameter $R$, heat source parameter $Q$ and Eckert number $E_{c}$ on temperature are depicted in Figure 17. Thermal boundary layer thickness increases with the increase in thermal radiation parameter $R$. It is noticed that an increase in heat generation parameter increases the thermal boundary layer thickness. This is owing to the fact that heat source can add more heat to the plate which increases its temperature. The Eckert number enunciates the ratio of the kinetic energy in the flow and the enthalpy. The positive Eckert number corresponds to the cooling plate i.e., the fluid can take heat from the plate. The thermal buoyancy force increases with the increase of radiation parameter $R$, heat source parameter $Q$ and Eckert number $E_{c}$ that means the rate of heat difference between the plate and the fluid decreases.

The effect of radiation absorption parameter $Q_{1}$ on velocity, temperature, concentration, Nusselt number and Sherwood number is illustrated in Figures 18-22. At the position of $Y=0.5$ the values of velocity are recorded as 1.65010 , $1.69753,1.74318,1.78711$ and 1.82938 for radiation absorption parameter $Q_{1}=$ 1, 2, 3, 4 and 5 respectively. From Figure 18 it is clearly observed that the maximum velocity increases by $4.828 \%$ for the change of radiation absorption parameter $Q_{1}$ from 4 to 5 which happen at same point $Y=0.5$. The temperatures are computed as $0.9352,1.04030,1.14076,1.23672$ and 1.32832 at $Y=1.0$ for radiation absorption parameter $Q_{1}=1,2,3,4$ and 5 respectively and the maximum temperature increases by $10.51 \%$. In Figure 20 the concentration increases by $2.977 \%, 2.847 \%, 2.723 \%$ and $2.601 \%$ as the changes of radiation absorption parameter $Q_{1}$ as follows 1 to 2,2 to 3,3 to 4 and 4 to 5 which occur at $Y=0.75$. The coefficients of Nusselt number decrease due to the increase of radiation absorption parameter. At the time $\tau=0.397$ the coefficients of Nusselt number are recorded as $0.04451,-0.02754,-0.09647,-0.16243$ and -0.22556 for radiation absorption parameter $Q_{1}=1,2,3,4$ and 5 respectively. Here we saw that the Nusselt number decreases with the increasing values of radiation absorption parameter $Q_{1}$. This result is similar with Vedavathi et al. [12], Prakash et al. [13] and Umamaheswar et al. [14].

Figure 23 shows the concentration profile for various values of Schmidt number $S_{c}$, Soret number $S_{r}$ and chemical reaction parameter $K_{c}$. Physically the 
rise of Schmidt number $S_{c}$ means a decrease of molecular diffusivity, which results in a decrease of the species boundary layer. The mass flux generated by temperature gradient is designate as the Soret effect. The concentration profile increases with the increasing vales of Soret number $S_{r}$ An increase in the chemical reaction parameter $K_{c}$ leads to the downfall of the concentration.

The effect of higher order chemical reaction $I$ on concentration profile is delimitated in Figure 24. From the Figure 24, it is seen that the concentration increases with increase of the order of chemical reaction 1 . The similar result is shown by Alam and Ahammad [32].

The effect of electrical conductivity parameter $\mathcal{\varepsilon}$ on the improvement of streamlines and isotherms are presented in Figure 25 and Figure 26. Velocity

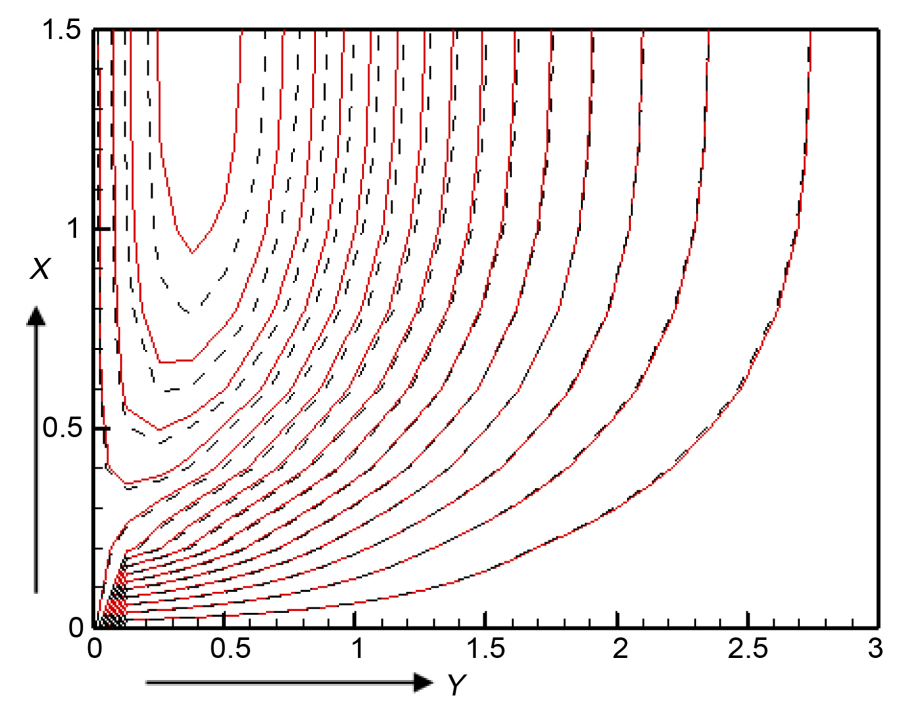

Figure 25. Streamlines for $\varepsilon=0$ (red solid line) and $\varepsilon=0.75$ (black dashed line).

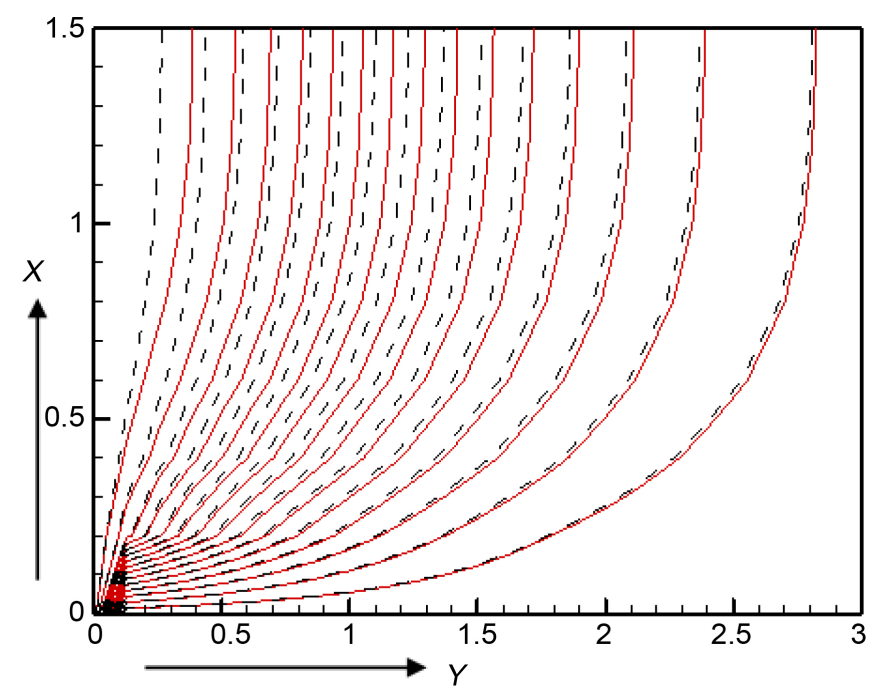

Figure 26. Isotherms for $\mathcal{E}=0$ (red solid line) and $\mathcal{E}=0.75$ (black dashed line). 
boundary layer increases and thermal boundary layer decreases owing to the increase of electrical conductivity parameter $\varepsilon$. Figure 27 and Figure 28 delimitate the significant effect of radiation absorption parameter $Q_{1}$ on streamlines and isotherms.

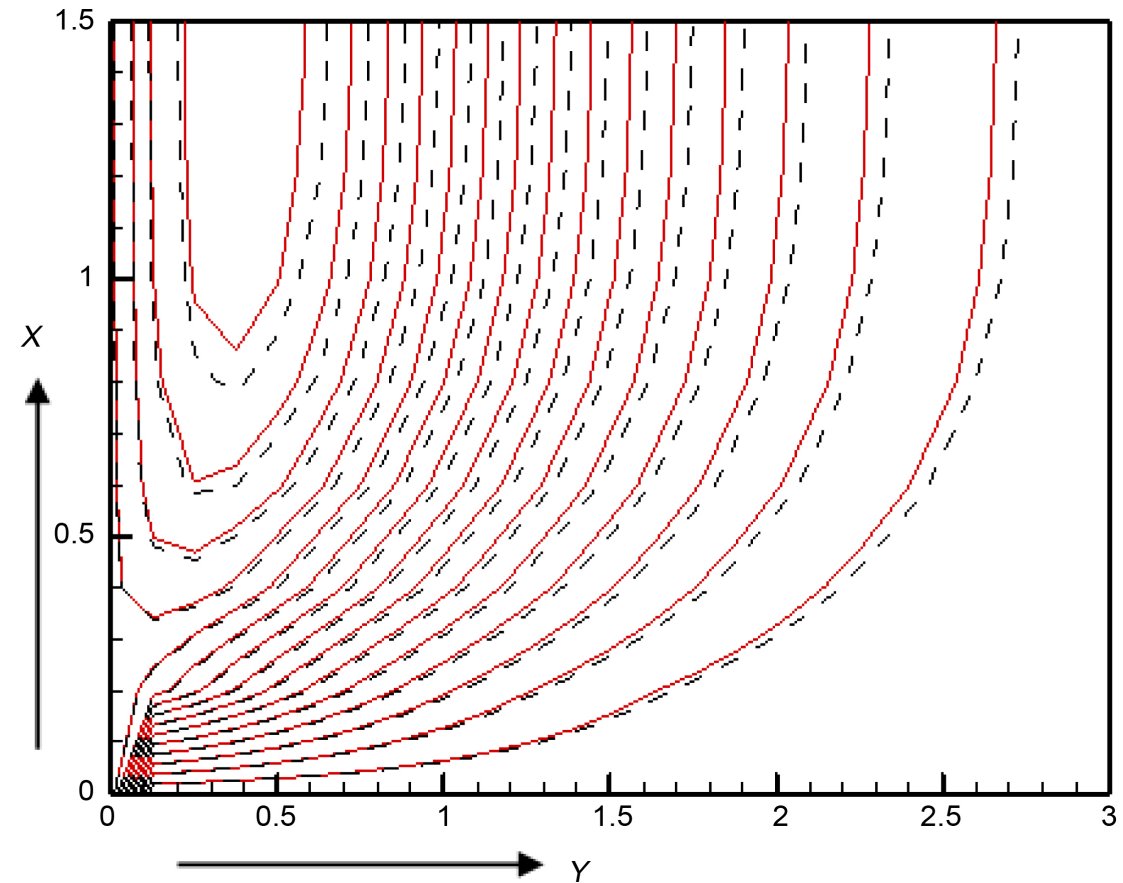

Figure 27. Streamlines for $Q_{1}=0$ (red solid line) and $Q_{1}=0.75$ (black dashed line).

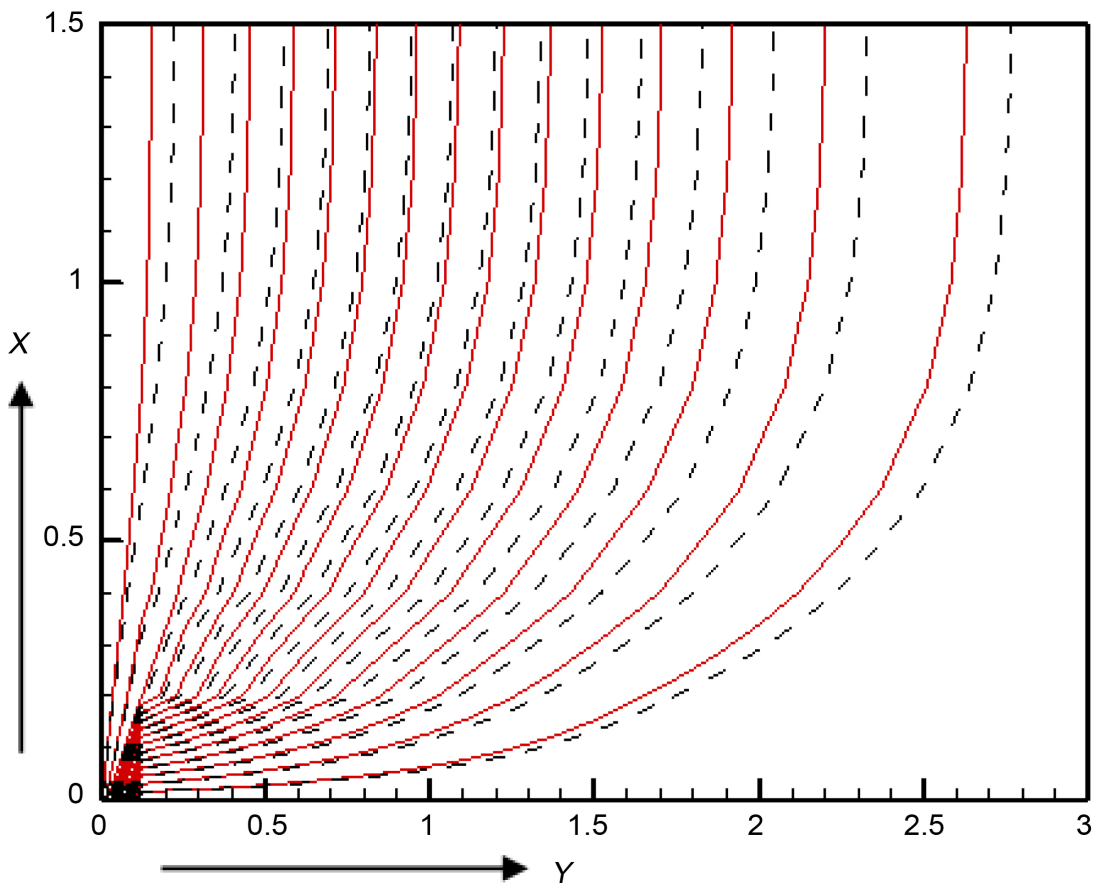

Figure 28. Isotherms for $Q_{1}=0$ (red solid line) and $Q_{1}=0.75$ (black dashed line). 


\section{Conclusions}

The study that we have carried out considered the effects of higher order chemical reaction, variable electrical conductivity on high-speed MHD past an exponential accelerated inclined plate. In our investigation, we have been analyzed stability criterion to make this experiment effortful. We have considered the chemical reaction as second order. Also, we have taken $P_{r}=0.71$ and $S_{c}=0.66$ in our computations. From the above investigation the following assumptions may be drawn:

- Due to the increase of inclination angle $\varphi$ velocity decreases.

- Velocity increases owing to the increase of acceleration parameter $a$.

- In the presence of high speed MHD the temperature profile increases.

- Electrical conductivity parameter $\mathcal{E}$ leads to an increase in velocity and skin friction whereas temperature and Nusselt number decreases.

- An increase in the Prandtl number $P_{r}$ implies decrease in the velocity and temperature and increase in the Nusselt number.

- Owing to increase the radiation absorption parameter $Q_{1}$ velocity, temperature and Sherwood number increase but the temperature of the fluid decreases.

- The Schmidt number $S_{c}$ is inversely proportional to the concentration which leads to a decrease in the concentration profiles.

The concentration profile increases with the increasing values of order of chemical reaction 1 .

\section{References}

[1] Shercliff, J.A. (1965) A Textbook of Magnetohydrodynamics. Pergamon Press, London.

[2] Ferraro, V.C.A. and Plumpton, C. (1966) An Introduction to Magneto Fluid Mechanics. Clarendon Press, Oxford.

[3] Jeffrey, A. (1966) Magnetohydrodynamics. Oliver and Boyd, Edinburgh.

[4] Pop, I. and Ingham, D.B. (2001) Convective Heat Transfer. Pergamon Press, London.

[5] Finston, M. (1956) Free Convection past a Vertical Plate. Journal of Applied Mathematical Physics, 7, 527-529. https://doi.org/10.1007/BF01601181

[6] Forchheimer, P. (1901) Wasserbewegung durch Boden. $45^{\text {th }}$ Edition, Zeitschrift des Vereins deutscher Ingenieure, Düsseldorf.

[7] Wooding, R. (1957) Steady State Free Thermal Convection of Liquid in a Saturated Permeable Medium. Journal of Fluid Mechanics, 2, 273-285. https://doi.org/10.1017/S0022112057000129

[8] Brickmam, H.C. (1947) A Calculation of the Viscous Force Exerted by a Flowing Fluid on a Dense Swarm of Particles. Applied Sciences Research, 1, 27-34.

[9] Brickman, H.C. (1947) On the Permeability of Media Consisting of Closely Packed Porous Particles. Applied Sciences Research, 1, 81-86.

https://doi.org/10.1007/BF02120318

[10] Raju, M.C., Reddy, N.A. and Varma, S.V.K. (2014) Analytical Study of MHD Free 
Convective, Dissipative Boundary Layer Flow past a Porous Vertical Surface in the Presence of Thermal Radiation, Chemical Reaction and Constant Suction. Ain Shams Engineering Journal, 5, 1361-1369. https://doi.org/10.1016/j.asej.2014.07.005

[11] Mondal, R.K., Hossain, M.A., Rana, B.M.J. and Ahmmed, S.F. (2015) Radiation and Chemical Reaction Effects on Free Convection and Mass Transfer Flow of Dissipative Fluid past an Infinite Vertical Plate through a Porous Medium. Elixir International Journal, 84, 523-530.

[12] Vedavathi, N., Ramakrishna, K. and Reddy, K.J. (2014) Radiation and Mass Transfer Effects on Unsteady MHD Convective Flow past an Infinite Vertical Plate with Dufour and Soret Effects. Ain Shams Engineering Journal, 6, 363-371.

https://doi.org/10.1016/j.asej.2014.09.009

[13] Prakash, J., Prasad, P.D., Kumar, R.V.M.S.S.K. and Varma, S.V.K. (2016) Diffusion-Thermo Effects on MHD Free Convective Radiative and Chemically Reactive Boundary Layer Flow through a Porous Medium over a Vertical Plate. Journal of Computational and Applied Research in Mechanical Engineering, 5, 111-126.

[14] Umamaheswar, M., Raju, M.C., Varma, S.V.K. and Gireeshkumar, J. (2016) Numerical Investigation of MHD Free Convection Flow of a Non-Newtonian Fluid past an Impulsively Started Vertical Plate in the Presence of Thermal Diffusion and Radiation Absorption. Alexandria Engineering Journal, 55, 2005-2014. https://doi.org/10.1016/j.aej.2016.07.014

[15] Desale, S.V. and Pradhan, V.H. (2013) Implicit Finite Difference Solution of MHD Boundary Layer Heat Transfer over a Moving Plate. IOSR Journal of Mathematics, 9, 18-23.

[16] Mishra, S.R. and Jena, S. (2014) Numerical Solution of Boundary Layer MHD Flow with Viscous Dissipation. The Scientific World Journal, 2014, 1-5. https://doi.org/10.1155/2014/756498

[17] Haque, M.M. and Sarder, U.K. (2015) Thermal Diffusion Effect on Convective Heat and Mass Transfer of High Speed MHD Flow over a Stretching Sheet. Journal of Scientific Research \& Reports, 8, 1-14. https://doi.org/10.9734/JSRR/2015/18075

[18] Einstein, A. (1906) Eineneue Bestinunung der Molekul dimension. Annals of Physics, 19, 286-306.

[19] Gebhar, B. (1962) Effects of Viscous Dissipation in Natural Convection. Journal of Fluid Mechanics, 14, 225-232. https://doi.org/10.1017/S0022112062001196

[20] Kishore, P.M., Rajesh, V. and Verma, S.V.K. (2010) Effects of Heat Transfer and Viscous Dissipation on MHD Free Convection Flow past an Exponentially Accelerated Vertical Plate with Variable Temperature. Journal of Naval Architecture and Marine Engineering, 7, 101-110.

[21] Sakiadis, B.C. (1961) Boundary-Layer Behavior on Continuous Solid Surfaces, AIChE Journal, 7, 26-28.

[22] Abd El-Naby, M.A., Elbarbary, E.M.E. and Abdelazem, N.Y. (2012) Finite Difference Solution of Radiation Effects on MHD Unsteady Free-Convection Flow over Vertical Plate with Variablesurface Temperature. Journal of Applied Mathematics, 2, 65-86.

[23] Rajput, U.S. and Kumar, S. (2012) Radiation Effects on MHD Flow past an Impulsively Started Vertical Plate with Variable Heat and Mass Transfer. International Journal of Applied Mathematics and Mechanics, 8, 66-85.

[24] Gundagani, M., Sheri, S., Paul, A. and Reddy, M.C.K. (2013) Unsteady Magnetohydrodynamics Free Convective Flow past a Vertical Porous Plate. International Journal of Applied Science and Engineering, 11, 267-275. 
[25] Javaherdeh, K., Nejad, MM. and Moslemi, M. (2015) Natural Convection Heat and Mass Transfer in MHD Fluid Flow past a Moving Vertical Plate with Variable Surface Temperature and Concentration in a Porous Medium. Engineering Science and Technology, an International Journal, 18, 423-431.

[26] Pattnaik, J.R., Dash, G.C. and Singh, S. (2017) Radiation and Mass Transfer Effects on MHD Flow through Porous Medium past an Exponentially Accelerated Inclined Plate with Variable Temperature. Ain Shams Engineering Journal, 8, 67-75. https://doi.org/10.1016/j.asej.2015.08.014

[27] Shrama, P.R. and Singh, G. (2010)Steady MHD Natural Convection Flow with Variable Electrical Conductivity and Heat Generation along an Isothermal Vertical Plate. Tamkang Journal of Science and Engineering, 13, 235-242.

[28] Chakraborty, S. and Medhi, N. (2016) MHD Convective Flow along a Vertical Isothermal Plate under Variable Electrical Conductivity and Heat Generation in Porous Medium. International Journal of Current Research, 8, 30374-30379.

[29] Sharma, S. and Deka, R.K. (2012) Chemical Reaction Effects on MHD Mixed Convection Flow of Water at Maximum Density past a Vertical Plate under Variable Temperature. IOSR Journal of Applied Physics, 1, 1-7.

[30] Sharma, B.K., Yadav, K., Mishra, N.K. and Chaudhary, R.C. (2012) Soret and Dufour Effects on Unsteady MHD Mixed Convection Flow past a Radiative Vertical Porous Plate Embedded in a Porous Medium with Chemical Reaction. Applied Mathematics, 3, 717-723. https://doi.org/10.4236/am.2012.37105

[31] Alam, M.S. and Ahammad, M.U. (2011) Effects of Variable Chemical Reaction and Variable Electric Conductivity on Free Convective Heat and Mass Transfer Flow along an Inclined Stretching Sheet with Variable Heat and Mass Fluxes under the Influence of Dufour and Soret Effects. Nonlinear Analysis: Modelling and Control, 16, 1-16.

[32] Kumar, B.R., Sivaraj, R. and Benazir, J. (2015) Chemically Reacting MHD Free Convective Flow over a Vertical Cone with Variable Electric Conductivity. International Journal of Pure and Applied Mathematics, 5, 821-828.

\section{Submit or recommend next manuscript to SCIRP and we will provide best service for you:}

Accepting pre-submission inquiries through Email, Facebook, LinkedIn, Twitter, etc. A wide selection of journals (inclusive of 9 subjects, more than 200 journals)

Providing 24-hour high-quality service

User-friendly online submission system

Fair and swift peer-review system

Efficient typesetting and proofreading procedure

Display of the result of downloads and visits, as well as the number of cited articles

Maximum dissemination of your research work

Submit your manuscript at: http://papersubmission.scirp.org/

Or contact wjm@scirp.org 